\title{
Opposite-parity contaminations in lattice nucleon form factors
}

\author{
Finn M. Stokes, ${ }^{1,2, *}$ Waseem Kamleh, ${ }^{1}$ and Derek B. Leinweber ${ }^{1}$ \\ ${ }^{1}$ Special Research Centre for the Subatomic Structure of Matter, Department of Physics, \\ University of Adelaide, South Australia 5005, Australia \\ ${ }^{2}$ Jülich Supercomputing Centre, Institute for Advanced Simulation, Forschungszentrum Jülich, \\ Jülich D-52425, Germany
}

(Received 8 October 2018; published 12 April 2019)

\begin{abstract}
The recently introduced parity expanded variational analysis (PEVA) technique allows for the isolation of baryon eigenstates at finite momentum free from opposite-parity contamination. In this paper, we establish the formalism for computing form factors of spin-1/2 states using PEVA. Selecting the vector current, we compare the electromagnetic form factors of the ground state nucleon extracted via this technique to a conventional parity-projection approach. Our results show a statistically significant discrepancy between the PEVA and conventional analyses. This indicates that existing calculations of matrix elements of ground state baryons at finite momentum can be affected by systematic errors of $~ 20 \%$ at physical quark masses. The formalism introduced here provides an effective approach to removing these systematic errors.
\end{abstract}

DOI: 10.1103/PhysRevD.99.074506

\section{INTRODUCTION}

Lattice QCD investigations of baryon matrix elements form a rich and varied field of study. In such investigations, it is necessary to isolate the state of interest from the tower of energy levels observed on the lattice. For ground states, this can be achieved through simple Euclidean time evolution, but it is common to use more advanced techniques such as multiexponential fits, the summation method, or variational analysis in order to extract the signal of interest from earlier time slices and avoid the noise present in the tails of correlation functions. Regardless of the specific technique used, when working with spin- $1 / 2$ baryons it is typical to perform a simple zero-momentum parity projection to the parity sector of interest, significantly reducing the number of possible contaminating states before even beginning the analysis. This technique works perfectly for at-rest baryons, completely removing opposite-parity contaminations, but for matrix elements where at least one of the initial or final state is boosted to nontrivial momentum this is no longer the case. Since eigenstates with nonzero momentum are not eigenstates of parity, the parity sectors are no longer well defined and a

\footnotetext{
*Corresponding author. f.stokes@fz-juelich.de

Published by the American Physical Society under the terms of the Creative Commons Attribution 4.0 International license. Further distribution of this work must maintain attribution to the author(s) and the published article's title, journal citation, and DOI. Funded by SCOAP ${ }^{3}$.
}

naïve parity projection allows opposite-parity states to reenter the correlation functions.

In Ref. [1], we introduced the parity expanded variational analysis (PEVA) technique to address this issue, applying it to the extraction of the nucleon spectrum from two point correlation functions. In this paper, we extend the formalism to the computation of matrix elements from three-point correlation functions, and apply it to the specific example of calculating the Sachs electric and magnetic form factors $G_{E}\left(Q^{2}\right)$ and $G_{M}\left(Q^{2}\right)$ of the ground-state proton and neutron. The Sachs form factors describe the response of a baryon to the vector current. At low $Q^{2}$, these form factors give information about the large-scale electromagnetic structure of the state, such as its charge radius and magnetic moment; at high $Q^{2}$ they give information about the short-distance internal structure of the state. These form factors can be determined experimentally to high accuracy. Computing them in ab-initio lattice QCD provides an important confrontation of theory with experiment.

In addition, computing these form factors on the lattice gives us important insight into the underlying physics. For example, on the lattice it is possible to separately compute the contributions to the form factors from connected diagrams (as studied in this paper) and disconnected diagrams, giving insight into the role sea quarks play in the structure of the proton and the neutron. One can also alter the electric charges of the quarks, readily illustrating the quark-flavor structure of the nucleon.

We probe the values of these form factors by creating an incoming nucleon on the lattice, having it interact with a vector current with some momentum transfer $\boldsymbol{q}$, and then 
annihilating the outgoing nucleon with a fixed momentum $\boldsymbol{p}^{\prime}$. By momentum conservation, the incoming state must have momentum $\boldsymbol{p}=\boldsymbol{p}^{\prime}-\boldsymbol{q}$. Due to the way we include the vector current on the lattice, we only consider a small number of fixed momentum transfers $\boldsymbol{q}$. By varying the three-momenta of the outgoing state and hence the incoming state, we gain access to the form factors at a range of

$$
Q^{2}=\boldsymbol{q}^{2}-\left(\sqrt{m_{N}^{2}+\boldsymbol{p}^{\prime 2}}-\sqrt{m_{N}^{2}+\boldsymbol{p}^{2}}\right)^{2} .
$$

In particular, these boosts provide access to values close to $Q^{2}=0$, well below $\left|\boldsymbol{q}_{\min }\right|^{2}=\left(2 \pi / L_{s}\right)^{2}$, without requiring the use of twisted boundary conditions. By accessing a range of values, we gain insight into the $Q^{2}$ dependence of the form factors, and can make a comparison with various models and experiment. By studying the low- $Q^{2}$ dependence of the electric form factor, we can make an $a b$-initio determination of the charge radius of the proton. In addition, we observe that when considering the contributions from each quark flavor independently, $G_{E}\left(Q^{2}\right)$ and $G_{M}\left(Q^{2}\right)$ have a similar $Q^{2}$ dependence in the range considered. Hence, we can access the magnetic dipole moments of the proton and neutron by taking ratios of the quark-sector form factors.

\section{PARITY EXPANDED VARIATIONAL ANALYSIS}

The process of extracting a baryonic excited-state spectrum via the PEVA technique is presented in full in Ref. [1]. We present here a brief summary of this process to introduce the notation and concepts necessary to describe the extension to the computation of baryonic matrix elements.

We begin with a basis of $n$ conventional spin- $1 / 2$ operators $\left\{\chi_{i}(x)\right\}$ that couple to the states of interest. Adopting the Pauli representation of the gamma matrices, we introduce the PEVA projector $\Gamma_{ \pm p} \equiv \frac{1}{4}\left(\mathbb{I}+\gamma^{4}\right)$ $\left(\mathbb{I} \pm i \gamma^{5} \gamma^{k} \hat{\boldsymbol{p}}^{k}\right)$. When acting on a spinor, $\Gamma_{ \pm \boldsymbol{p}}$ projects to states of definite helicity. The choice of sign corresponds to choosing the sign of the projected helicity. For two point correlation functions, this choice of helicity is arbitrary, and both formulations of the projector give the same results.

We construct a set of basis operators

$$
\begin{gathered}
\chi_{ \pm p i}(x) \equiv \Gamma_{ \pm p} \chi_{i}(x), \\
\chi_{ \pm p i^{\prime}}(x) \equiv \pm \Gamma_{ \pm p} \gamma^{5} \chi_{i}(x) .
\end{gathered}
$$

We note that we use a Euclidean metric $\delta^{\mu \nu}$, and hence there is no need to distinguish between contravariant and covariant indices.

As described in Ref. [2], three-dimensional smearing of the operators breaks Lorentz invariance, and as a result can alter their transformation properties, introducing extra terms into the operator coupling proportional to $\gamma^{4}$. However, due to the structure of the PEVA projector, $\Gamma_{ \pm p} \gamma^{4}=\Gamma_{ \pm p}$. As a result, the additional Dirac structure introduced is removed by the PEVA projection. The only remaining effect is that due to the factor of $\gamma^{5}$ introduced for the primed operator, the primed and unprimed operators may have different couplings to each state. In the absence of three-dimensional smearing they differ only by kinematic factors. Hence the PEVA technique effectively handles the nontrivial Dirac structure arising from three-dimensional smearing, without the requirement for any special treatment.

We then seek an optimized set of operators $\phi_{ \pm p \alpha}(x)$ that each couple strongly to a single energy eigenstate $\alpha$. These optimized operators are constructed as linear combinations of the basis operators

$$
\begin{aligned}
& \phi_{ \pm \boldsymbol{p} \alpha}(x) \equiv \sum_{i} v_{\alpha i}(\boldsymbol{p}) \chi_{ \pm \boldsymbol{p} i}(x), \\
& \bar{\phi}_{ \pm \boldsymbol{p} \alpha}(x) \equiv \sum_{i} u_{\alpha i}(\boldsymbol{p}) \bar{\chi}_{ \pm \boldsymbol{p} i}(x),
\end{aligned}
$$

where the sum is over both the primed and unprimed operators. The coefficients $v_{\alpha i}(\boldsymbol{p})$ and $u_{\alpha i}(\boldsymbol{p})$ can be found as the left and right generalized eigenvectors [3,4] of $G(\boldsymbol{p} ; t+\Delta t)$ and $G(\boldsymbol{p} ; t)$, where the correlation matrix

$$
G_{i j}(\boldsymbol{p} ; t) \equiv \operatorname{Tr}\left(\sum_{\boldsymbol{x}} \mathrm{e}^{-i \boldsymbol{p} \cdot \boldsymbol{x}}\left\langle\Omega\left|\chi_{ \pm \boldsymbol{p} i}(x) \bar{\chi}_{ \pm \boldsymbol{p} i}(0)\right| \Omega\right\rangle\right),
$$

with $i$ and $j$ ranging over both the primed and unprimed operators.

The choice of sign in the PEVA projector has no effect on the values of these two-point correlation functions. This follows from the Dirac structure of the baryons and the standard trace properties of the associated gamma matrices. We note that the basis operators occur twice in the correlation function expression, for both creation and annihilation. In the top-left block, both operators are unprimed and the contributions from the cross-parity term in the projector $\left( \pm i \gamma^{5} \gamma^{k} \hat{\boldsymbol{p}}^{k}\right)$ are zero, so the overall sign is consistent, regardless of the choice of $\Gamma_{p}$ and $\Gamma_{-p}$. In the bottom-right block, both operators are primed, so two factors of \pm 1 are introduced by the overall sign of $\chi_{ \pm \boldsymbol{p} i^{\prime}}(x)$ in Eq. (2b), and the contributions from the cross-parity term are once again zero, so the factors of \pm 1 cancel and the overall sign is consistent. In the off-diagonal blocks, the contributions to the correlator are only from the cross-parity term, so there is an overall factor of \pm 1 , which cancels with the factor of \pm 1 from the single primed operator in each of these blocks. Hence, the values of the two-point correlation functions will be the same regardless of the choice of $\Gamma_{p}$ or $\Gamma_{-p}$. As a result, the coefficients for constructing $\phi_{+p \alpha}(x)$ and $\phi_{-p \alpha}(x)$ are identical, up to a choice of overall sign of the eigenvector. We choose the eigenvector sign to ensure the operators match at zero momentum, where the choice of $\pm \boldsymbol{p}$ has no effect on the physics. 
Using the optimized operators, we can construct the eigenstate-projected two-point correlation function

$$
\begin{aligned}
G(\boldsymbol{p} ; t ; \alpha) & \equiv \operatorname{Tr}\left(\sum_{\boldsymbol{x}} \mathrm{e}^{-i \boldsymbol{p} \cdot \boldsymbol{x}}\left\langle\Omega\left|\phi_{ \pm \boldsymbol{p} \alpha}(x) \bar{\phi}_{ \pm \boldsymbol{p} \alpha}(0)\right| \Omega\right\rangle\right) \\
& =v_{\alpha i}(\boldsymbol{p}) G_{i j}(\boldsymbol{p} ; t) u_{\alpha j}(\boldsymbol{p}) .
\end{aligned}
$$

\section{BARYON MATRIX ELEMENTS}

\section{A. General matrix elements}

In this section, we establish the formalism to extend the PEVA technique to the computation of baryon form factors. To perform the extension, we consider three-point correlation functions, inspecting their Dirac structure to extract the signal of interest. We then take ratios with two point functions to remove the time dependence and cancel out dependence on the interpolator couplings. The calculations are performed in the most general kinematics that can be realized.

Due to a lattice Ward identity associated with the conserved current, the three-point correlation functions for the electric form factor normalized to unit charge must approach the two-point correlation functions exactly on a configuration-by-configuration basis as $Q^{2} \rightarrow 0$. As a result, the two- and three-point correlation functions are highly correlated at low $Q^{2}$. The ratios we take facilitate the cancellation of statistical fluctuations, significantly reducing the statistical uncertainties in our extracted form factors.

By performing a parity-expanded variational analysis as described in Sec. II, we construct optimized operators $\phi_{ \pm p \alpha}(x)$ that couple to each state $\alpha$. We can use these operators to calculate the three point correlation functions

$$
\begin{aligned}
\mathcal{G}_{ \pm}^{3}\left(\mathcal{J} ; \boldsymbol{p}^{\prime}, \boldsymbol{p} ; t_{2}, t_{1} ; \alpha\right) \equiv & \sum_{\boldsymbol{x}_{2}, \boldsymbol{x}_{2}} \mathrm{e}^{-i \boldsymbol{p}^{\prime} \cdot \boldsymbol{x}_{2}} \mathrm{e}^{i\left(\boldsymbol{p}^{\prime}-\boldsymbol{p}\right) \cdot \boldsymbol{x}_{1}} \\
& \times\left\langle\Omega\left|\phi_{ \pm \boldsymbol{p}^{\prime} \alpha}\left(x_{2}\right) \mathcal{J}\left(x_{1}\right) \overline{\boldsymbol{\phi}}_{+\boldsymbol{p} \alpha}(0)\right| \Omega\right\rangle,
\end{aligned}
$$

where $\mathcal{J}(x)$ is some current operator, which is inserted with a momentum transfer $\boldsymbol{q}=\boldsymbol{p}^{\prime}-\boldsymbol{p}$. The consideration of $\mathcal{G}_{-}^{3}\left(\mathcal{J} ; \boldsymbol{p}^{\prime}, \boldsymbol{p} ; t_{2}, t_{1} ; \alpha\right)$ (where the sink operator uses the opposite PEVA projector sign convention to the source operator) is required to optimize the extraction of the form factors for general kinematics. We note that it is sufficient to consider this change of projector for the sink operator alone, leaving the source operator as $\bar{\phi}_{+p \alpha}(0)$ in all cases considered.

By inserting the complete set of states

$$
\mathbb{I}=\sum_{B, p, s}|B ; p ; s\rangle\langle B ; p ; s|
$$

on either side of the current, and noting the use of Euclidean time and fixed boundary conditions (or negligible backward-running state contributions), we can rewrite this three point correlation function as

$$
\begin{aligned}
\mathcal{G}_{ \pm}^{3}\left(\mathcal{J} ; \boldsymbol{p}^{\prime}, \boldsymbol{p} ; t_{2}, t_{1} ; \alpha\right)= & \sum_{s^{\prime}, s} \mathrm{e}^{-E_{\alpha}(\boldsymbol{p}) t_{1}} \mathrm{e}^{-E_{\alpha}\left(\boldsymbol{p}^{\prime}\right)\left(t_{2}-t_{1}\right)} \\
& \times\left\langle\Omega\left|\phi_{ \pm \boldsymbol{p}^{\prime} \alpha}(0)\right| \alpha ; p^{\prime} ; s^{\prime}\right\rangle \\
& \times\left\langle\alpha ; p^{\prime} ; s^{\prime}|\mathcal{J}(0)| \alpha ; p ; s\right\rangle \\
& \times\left\langle\alpha ; p ; s\left|\bar{\phi}_{\boldsymbol{p} \alpha}(0)\right| \Omega\right\rangle
\end{aligned}
$$

Note, the formalism presented here assumes perfect state isolation such that each optimized operator couples only to a single state.

We see that the time dependence of this three point correlator is entirely contained within exponentials of the energy, and the remaining structure depends on both the overlap of the optimized operator with its corresponding state

$$
\left\langle\Omega\left|\phi_{ \pm \boldsymbol{p} \alpha}(0)\right| \alpha ; p ; s\right\rangle=z_{\boldsymbol{p} \alpha} \sqrt{\frac{m_{\alpha}}{E_{\alpha}(\boldsymbol{p})}} \Gamma_{ \pm p} u_{\alpha}(p, s),
$$

and the matrix element for the current operator, $\left\langle\alpha ; p^{\prime}\right.$; $\left.s^{\prime}|\mathcal{J}(0)| \alpha ; p ; s\right\rangle$.

As we will see below, this Euclidean time dependence, along with the scalar factors relating to the coupling between the optimized operator and the state $\alpha$ can be removed by taking appropriate ratios with the two point correlation functions

$$
\begin{aligned}
G(\boldsymbol{p} ; t ; \alpha)= & \operatorname{Tr}\left(\sum_{s} \mathrm{e}^{-E_{\alpha}(\boldsymbol{p}) t}\left\langle\Omega\left|\phi_{ \pm \boldsymbol{p} \alpha}(0)\right| \alpha ; p ; s\right\rangle\right. \\
& \left.\times\left\langle\alpha ; p ; s\left|\bar{\phi}_{ \pm \boldsymbol{p} \alpha}(0)\right| \Omega\right\rangle\right) .
\end{aligned}
$$

\section{B. Vector current matrix elements}

In this paper, we investigate the electromagnetic properties of the proton and neutron by choosing the current operator $\mathcal{J}(x)$ to be the vector current. In particular, we use the tree-level $O(a)$-improved [5] conserved vector current used in Ref. [6],

$$
j_{C I}^{\mu}(x) \equiv j_{C}^{\mu}(x)+\frac{r}{2} a \bar{q}(x)\left(\stackrel{\nabla}{\nabla}^{\rho}+\vec{\nabla}^{\rho}\right) \sigma^{\rho \mu} q(x),
$$

where $r$ is the Wilson parameter, and

$\vec{\nabla}^{\mu} q(x) \equiv \frac{1}{2}\left(U^{\mu}(x) q\left(x+e^{\mu}\right)-U^{\dagger \mu}\left(x-e^{\mu}\right) q\left(x-e^{\mu}\right)\right)$

$\bar{q}(x) \overleftarrow{\nabla}^{\mu} \equiv \frac{1}{2}\left(\bar{q}\left(x+e^{\mu}\right) U^{\dagger \mu}(x)-\bar{q}\left(x-e^{\mu}\right) U^{\mu}\left(x-e^{\mu}\right)\right)$. 
This current is derived from the standard conserved vector current for the Wilson action, symmetrized around a lattice site

$$
\begin{aligned}
j_{C}^{\mu}(x) \equiv & \frac{1}{4}\left[\bar{q}(x)\left(\gamma^{\mu}-r\right) U^{\mu}(x) q\left(x+e^{\mu}\right)\right. \\
& +\bar{q}\left(x+e^{\mu}\right)\left(\gamma^{\mu}+r\right) U^{\dagger \mu}(x) q(x) \\
& +\bar{q}\left(x-e^{\mu}\right)\left(\gamma^{\mu}-r\right) U^{\mu}\left(x-e^{\mu}\right) q(x) \\
& \left.+\bar{q}(x)\left(\gamma^{\mu}+r\right) U^{\dagger \mu}\left(x-e^{\mu}\right) q\left(x-e^{\mu}\right)\right] .
\end{aligned}
$$

As all terms in the improved conserved current are onelink terms, the corrections to the tree-level couplings do not encounter large nonperturbative mean-field improvement corrections associated with tadpole contributions.

This choice of current operator gives the matrix element

$$
\begin{aligned}
& \left\langle\alpha ; p^{\prime} ; s^{\prime}\left|j_{C I}^{\mu}(0)\right| \alpha ; p ; s\right\rangle \\
& =\sqrt{\frac{m_{\alpha}}{E_{\alpha}(\boldsymbol{p})}} \sqrt{\frac{m_{\alpha}}{E_{\alpha}\left(\boldsymbol{p}^{\prime}\right)}} \bar{u}_{\alpha}\left(p^{\prime}, s^{\prime}\right) \\
& \quad \times\left(\gamma^{\mu} F_{1 \alpha}\left(Q^{2}\right)-\frac{\sigma^{\mu \nu} q^{\nu}}{2 m_{\alpha}} F_{2 \alpha}\left(Q^{2}\right)\right) \\
& \quad \times u_{\alpha}(p, s)
\end{aligned}
$$

where $Q^{2}=\boldsymbol{q}^{2}-\left(E_{\alpha}\left(\boldsymbol{p}^{\prime}\right)-E_{\alpha}(\boldsymbol{p})\right)^{2}$ is the squared fourmomentum with the conventional sign, and the invariant scalar functions $F_{1}\left(Q^{2}\right)$ and $F_{2}\left(Q^{2}\right)$ are respectively the Dirac and Pauli form factors.

Hence, using Eqs. (9) and (14) we can rewrite the correlator as

$$
\begin{aligned}
& \mathcal{G}_{ \pm}^{3}\left(j_{C I}^{\mu} ; \boldsymbol{p}^{\prime}, \boldsymbol{p} ; t_{2}, t_{1} ; \alpha\right) \\
& =\sum_{s^{\prime}, s} \mathrm{e}^{-E_{\alpha}(\boldsymbol{p}) t_{1}} \mathrm{e}^{-E_{\alpha}\left(\boldsymbol{p}^{\prime}\right)\left(t_{2}-t_{1}\right)} \frac{m_{\alpha}}{E_{\alpha}(\boldsymbol{p})} \frac{m_{\alpha}}{E_{\alpha}\left(\boldsymbol{p}^{\prime}\right)} z_{\boldsymbol{p}^{\prime} \alpha} \bar{z}_{\boldsymbol{p} \alpha} \\
& \quad \times \Gamma_{ \pm p^{\prime}} u_{\alpha}\left(p^{\prime}, s^{\prime}\right) \bar{u}_{\alpha}\left(p^{\prime}, s^{\prime}\right) \\
& \quad \times\left(\gamma^{\mu} F_{1 \alpha}\left(Q^{2}\right)-\frac{\sigma^{\mu \nu} q^{\nu}}{2 m_{\alpha}} F_{2 \alpha}\left(Q^{2}\right)\right) \\
& \quad \times u_{\alpha}(p, s) \bar{u}_{\alpha}(p, s) \Gamma_{\boldsymbol{p}} .
\end{aligned}
$$

Using the spin sum

$$
\sum_{s} u_{B}(p, s) \bar{u}_{B}(p, s)=\frac{-i \gamma \cdot p+m_{B}}{2 m_{B}}
$$

the three-point function is

$$
\begin{aligned}
\mathcal{G}_{ \pm}^{3} & \left(j_{C I}^{\mu} ; \boldsymbol{p}^{\prime}, \boldsymbol{p} ; t_{2}, t_{1} ; \alpha\right) \\
= & \mathrm{e}^{-E_{\alpha}(\boldsymbol{p}) t_{1}} \mathrm{e}^{-E_{\alpha}\left(\boldsymbol{p}^{\prime}\right)\left(t_{2}-t_{1}\right)} z_{\boldsymbol{p}^{\prime} \alpha} \bar{z}_{\boldsymbol{p} \alpha} \\
& \times \Gamma_{ \pm \boldsymbol{p}^{\prime}} \frac{-i \gamma \cdot p^{\prime}+m_{\alpha}}{2 E_{\alpha}\left(\boldsymbol{p}^{\prime}\right)}\left(\gamma^{\mu} F_{1 \alpha}\left(Q^{2}\right)-\frac{\sigma^{\mu \nu} q^{\nu}}{2 m_{\alpha}} F_{2 \alpha}\left(Q^{2}\right)\right) \\
& \times \frac{-i \gamma \cdot p+m_{\alpha}}{2 E_{\alpha}(\boldsymbol{p})} \Gamma_{\boldsymbol{p}} .
\end{aligned}
$$

To extract our desired signal from this spinor structure, we can take the spinor trace with some spin-structure projector $\Gamma_{S}$. This trace is then called the spinor-projected three-point correlation function

$$
\begin{aligned}
G_{ \pm}^{3}\left(\Gamma_{S} ; j_{C I}^{\mu} ; \boldsymbol{p}^{\prime}, \boldsymbol{p} ; t_{2}, t_{1} ; \alpha\right) \equiv & \operatorname{Tr}\left(\Gamma_{S} \mathcal{G}_{ \pm}^{3}\left(j_{C I}^{\mu} ; \boldsymbol{p}^{\prime}, \boldsymbol{p} ; t_{2}, t_{1} ; \alpha\right)\right) \\
= & \mathrm{e}^{-E_{\alpha}(\boldsymbol{p}) t_{1}} \mathrm{e}^{-E_{\alpha}\left(\boldsymbol{p}^{\prime}\right)\left(t_{2}-t_{1}\right)} z_{\boldsymbol{p}^{\prime} \alpha} \bar{z}_{\boldsymbol{p} \alpha}\left(\operatorname{Tr}\left(\Gamma_{S} \Gamma_{ \pm \boldsymbol{p}^{\prime}} \frac{-i \gamma \cdot p^{\prime}+m_{\alpha}}{2 E_{\alpha}\left(\boldsymbol{p}^{\prime}\right)} \gamma^{\mu} \frac{-i \gamma \cdot p+m_{\alpha}}{2 E_{\alpha}(\boldsymbol{p})} \Gamma_{\boldsymbol{p}}\right) F_{1 \alpha}\left(Q^{2}\right)\right. \\
& \left.-\operatorname{Tr}\left(\Gamma_{S} \Gamma_{ \pm \boldsymbol{p}^{\prime}} \frac{-i \gamma \cdot p^{\prime}+m_{\alpha}}{2 E_{\alpha}\left(\boldsymbol{p}^{\prime}\right)} \frac{\sigma^{\mu \nu} q^{\nu}}{2 m_{\alpha}} \frac{-i \gamma \cdot p+m_{\alpha}}{2 E_{\alpha}(\boldsymbol{p})} \Gamma_{\boldsymbol{p}}\right) F_{2 \alpha}\left(Q^{2}\right)\right) .
\end{aligned}
$$

If we consider the function

$$
F_{ \pm}^{\prime}\left(\Gamma_{S}, \mathcal{J}\right) \equiv 8 E_{\alpha}(\boldsymbol{p}) E_{\alpha}\left(\boldsymbol{p}^{\prime}\right) \operatorname{Tr}\left(\Gamma_{S} \Gamma_{ \pm \boldsymbol{p}^{\prime}} \frac{-i \gamma \cdot p^{\prime}+m_{\alpha}}{2 E_{\alpha}\left(\boldsymbol{p}^{\prime}\right)} \mathcal{J} \frac{-i \gamma \cdot p+m_{\alpha}}{2 E_{\alpha}(\boldsymbol{p})} \Gamma_{\boldsymbol{p}}\right),
$$

where the prime on $F_{ \pm}^{\prime}\left(\Gamma_{S}, \mathcal{J}\right)$ denotes the presence of the PEVA projectors, then we can express Eq. (18) as

$$
\begin{aligned}
G_{ \pm}^{3}\left(\Gamma_{S} ; j_{C I}^{\mu} ; \boldsymbol{p}^{\prime}, \boldsymbol{p} ; t_{2}, t_{1} ; \alpha\right)= & \mathrm{e}^{-E_{\alpha}(\boldsymbol{p}) t_{1}} \mathrm{e}^{-E_{\alpha}\left(\boldsymbol{p}^{\prime}\right)\left(t_{2}-t_{1}\right)} z_{\boldsymbol{p} \alpha} \bar{z}_{\boldsymbol{p} \alpha} \frac{1}{8 E_{\alpha}(\boldsymbol{p}) E_{\alpha}\left(\boldsymbol{p}^{\prime}\right)} \\
& \times\left(F_{ \pm}^{\prime}\left(\Gamma_{S}, \gamma^{\mu}\right) F_{1 \alpha}\left(Q^{2}\right)-\frac{q^{\nu}}{2 m_{\alpha}} F_{ \pm}^{\prime}\left(\Gamma_{S}, \sigma^{\mu \nu}\right) F_{2 \alpha}\left(Q^{2}\right)\right) .
\end{aligned}
$$

These spinor-projected correlation functions have a nontrivial time dependence, which can be removed by constructing the ratio [7] 


$$
\begin{aligned}
R_{ \pm}\left(\boldsymbol{p}^{\prime}, \boldsymbol{p} ; \alpha ; r, s\right) \equiv & \sqrt{\left|\frac{r^{\mu} G_{ \pm}^{3}\left(s^{\nu} \Gamma^{\nu} ; j_{C I}^{\mu} ; \boldsymbol{p}^{\prime}, \boldsymbol{p} ; t_{2}, t_{1} ; \alpha\right) r^{\rho} G_{ \pm}^{3}\left(s^{\sigma} \Gamma^{\sigma} ; j_{C I}^{\rho} ; \boldsymbol{p}, \boldsymbol{p}^{\prime} ; t_{2}, t_{1} ; \alpha\right)}{G\left(\boldsymbol{p}^{\prime} ; t_{2} ; \alpha\right) G\left(\boldsymbol{p} ; t_{2} ; \alpha\right)}\right|} \\
& \times \operatorname{sign}\left(r^{\lambda} G_{ \pm}^{3}\left(s^{\eta} \Gamma^{\eta} ; j_{C I}^{\lambda} ; \boldsymbol{p}^{\prime}, \boldsymbol{p} ; t_{2}, t_{1} ; \alpha\right)\right),
\end{aligned}
$$

where $\Gamma^{4}=\left(\mathbb{I}+\gamma^{4}\right) / 2$ and $\Gamma^{k}=\left(\mathbb{I}+\gamma^{4}\right)\left(i \gamma^{5} \gamma^{k}\right) / 2$ form the basis for the spin projectors we use, and $r^{\mu}$ and $s^{\mu}$ are coefficients selected to determine the form factors. Care is taken in selecting $r^{\mu}$ and $s^{\mu}$ to ensure that the relevant values of $F_{ \pm}^{\prime}\left(\Gamma_{S}, \mathcal{J}\right)$ remain purely real.

In addition, as the momentum transfer $\boldsymbol{q} \rightarrow \mathbf{0}$, charge conservation requires that the temporal component of the three point correlator for the conserved vector current becomes exactly proportional to the two point correlator on each gauge field configuration, that is

$$
G_{ \pm}^{3}\left(s^{\nu} \Gamma^{\nu} ; j_{C l}^{4} ; \boldsymbol{p}, \boldsymbol{p} ; t_{2}, t_{1} ; \alpha\right) \propto \mathcal{G}\left(\boldsymbol{p} ; t_{2} ; \alpha\right) .
$$

Because of this, taking the ratio in Eq. (21) facilitates the cancellation of statistical fluctuations in the two- and threepoint correlators, providing results with small statistical uncertainties, at least in the case of $G_{E}\left(Q^{2}\right)$.

We can then define the reduced ratio,

$$
\begin{aligned}
\bar{R}_{ \pm}\left(\boldsymbol{p}^{\prime}, \boldsymbol{p} ; \alpha ; r, s\right) \equiv & \sqrt{\frac{2 E_{\alpha}(\boldsymbol{p})}{E_{\alpha}(\boldsymbol{p})+m_{\alpha}}} \sqrt{\frac{2 E_{\alpha}\left(\boldsymbol{p}^{\prime}\right)}{E_{\alpha}\left(\boldsymbol{p}^{\prime}\right)+m_{\alpha}}} \\
& \times R_{ \pm}\left(\boldsymbol{p}^{\prime}, \boldsymbol{p} ; \alpha ; r, s\right) .
\end{aligned}
$$

Taking this reduced ratio and substituting in the expressions for the projected correlation functions, we obtain

$$
\begin{aligned}
\bar{R}_{ \pm}\left(\boldsymbol{p}^{\prime}, \boldsymbol{p} ; \alpha ; r, s\right) & =\frac{r^{\mu} s^{\nu}}{16 E_{\alpha}(\boldsymbol{p}) E_{\alpha}\left(\boldsymbol{p}^{\prime}\right)\left(E_{\alpha}(\boldsymbol{p})+m_{\alpha}\right)\left(E_{\alpha}\left(\boldsymbol{p}^{\prime}\right)+m_{\alpha}\right)} \\
& \times\left(F_{ \pm}^{\prime}\left(\Gamma^{\nu}, \gamma^{\mu}\right) F_{1 \alpha}\left(Q^{2}\right)\right. \\
& \left.-\frac{q^{\rho}}{2 m_{\alpha}} F_{ \pm}^{\prime}\left(\Gamma^{\nu}, \sigma^{\mu \rho}\right) F_{2 \alpha}\left(Q^{2}\right)\right)
\end{aligned}
$$

By investigating the $r^{\mu}$ and $s^{\sigma}$ dependence of this ratio, we find that the clearest signals are given by

$$
\begin{aligned}
& R_{ \pm}^{T}=\frac{2}{1 \pm \hat{\boldsymbol{p}} \cdot \hat{\boldsymbol{p}}^{\prime}} \bar{R}_{ \pm}\left(\boldsymbol{p}^{\prime}, \boldsymbol{p} ; \alpha ;(1, \mathbf{0}),(1, \mathbf{0})\right), \\
& R_{\mp}^{S}=\frac{2}{1 \pm \hat{\boldsymbol{p}} \cdot \hat{\boldsymbol{p}}^{\prime}} \bar{R}_{\mp}\left(\boldsymbol{p}^{\prime}, \boldsymbol{p} ; \alpha ;(0, \hat{\boldsymbol{r}}),(0, \hat{\boldsymbol{s}})\right),
\end{aligned}
$$

where $\hat{\boldsymbol{s}}$ is chosen such that $\boldsymbol{p} \cdot \hat{\boldsymbol{s}}=0=\boldsymbol{p}^{\prime} \cdot \hat{\boldsymbol{s}}, \hat{\boldsymbol{r}}$ is equal to $\hat{\boldsymbol{q}} \times \hat{\boldsymbol{s}}$, and the sign \pm in Eq. (25) is chosen such that $1 \pm$ $\hat{\boldsymbol{p}} \cdot \hat{\boldsymbol{p}}^{\prime}$ is maximized. This choice maximizes the signal in the lattice determination of the correlation function ratios.
We can then find the Sachs electric and magnetic form factors,

$$
\begin{gathered}
G_{E \alpha}\left(Q^{2}\right) \equiv F_{1 \alpha}\left(Q^{2}\right)-\frac{Q^{2}}{\left(2 m_{\alpha}\right)^{2}} F_{2 \alpha}\left(Q^{2}\right), \\
G_{M \alpha}\left(Q^{2}\right) \equiv F_{1 \alpha}\left(Q^{2}\right)+F_{2 \alpha}\left(Q^{2}\right),
\end{gathered}
$$

through appropriate linear combinations of $R_{ \pm}^{T}$ and $R_{\mp}^{S}$. A similar procedure can be applied to extract the relevant form factors from any current.

We have shown how the PEVA technique can be applied to the calculation of baryon form factors for arbitrary kinematics. Doing so ensures that these form factors are free from opposite parity contaminations, up to residual contaminations arising from the use of a finite operator basis.

\section{SACHS ELECTRIC FORM FACTOR}

We now apply this technique to calculate the Sachs electric form factors of the proton and the neutron. This gives us insight into the distribution of charge within these states.

The results in this paper are calculated on the PACS-CS $(2+1)$-flavor full-QCD ensembles [8], made available through the ILDG [9]. These ensembles use a $32^{3} \times 64$ lattice, and employ a renormalization-group improved Iwasaki gauge action with $\beta=1.90$ and nonperturbatively $O(a)$-improved Wilson quarks, with $C_{S W}=1.715$. We use five ensembles, with stated pion masses from $m_{\pi}=$ $702 \mathrm{MeV}$ to $156 \mathrm{MeV}$ [8], and set the scale using the Sommer parameter with $r_{0}=0.4921(64) \mathrm{fm}$ [8]. More details of the individual ensembles are presented in Table I, including the squared pion masses in the Sommer scale.

TABLE I. Details of the gauge field ensembles used in this analysis. For each ensemble we list both the pion mass given in Ref. [8], with the lattice spacing set by hadronic inputs, and our determination of the squared pion mass with the lattice spacing listed in the table, which is set by the Sommer parameter with $r_{0}=0.4921(64) \mathrm{fm}$ [8].

\begin{tabular}{lclcc}
\hline \hline PACS-CS & & & & \# src \\
$m_{\pi} / \mathrm{MeV}$ & $a / \mathrm{fm}$ & $m_{\pi}^{2} / \mathrm{GeV}$ & $\#$ conf. & \begin{tabular}{c} 
per conf. \\
\hline 702
\end{tabular} \\
570 & $0.1022(15)$ & $0.3884(113)$ & 399 & 1 \\
411 & $0.1009(15)$ & $0.2654(81)$ & 397 & 1 \\
296 & $0.0961(13)$ & $0.1525(43)$ & 449 & 2 \\
156 & $0.0933(13)$ & $0.0784(25)$ & 400 & 2 \\
\hline \hline
\end{tabular}


In these finite volumes, the momentum is quantized in units of $\left|\boldsymbol{q}_{\min }\right| \equiv \frac{2 \pi}{32 a}$. When fitting correlators, the $\chi^{2} /$ dof is calculated with the full covariance matrix, and the $\chi^{2}$ values of all fits are consistent with an appropriate $\chi^{2}$ distribution, as determined by a one-sided Kolmogorov-Smirnov test comparing the full set of all $\chi^{2}$ values for each number of degrees of freedom to the corresponding $\chi^{2}$ distribution.

The relativistic components of the baryon spinor are suppressed by the inverse of the baryon mass. Across the pion masses considered here, the nucleon mass ranges from 1.418(9) GeV to 0.993(15) GeV. As such, at the lighter pion masses, the relativistic components of the baryon spinor will be enhanced by a factor of $\sim 1.5$. As a result, the paritymixing at finite momentum will be increasingly problematic. However, at lighter pion masses, the gauge noise is more significant, and can occlude the parity-mixing effects if the statistics are insufficient.

For the variational analyses in this paper, we begin with an eight-interpolator basis is formed from the conventional spin- $1 / 2$ nucleon interpolators

$$
\begin{aligned}
& \chi_{1}=\epsilon^{a b c}\left[u^{a \top}\left(C \gamma^{5}\right) d^{b}\right] u^{c}, \quad \text { and } \\
& \chi_{2}=\epsilon^{a b c}\left[u^{a \top}(C) d^{b}\right] \gamma^{5} u^{c},
\end{aligned}
$$

with 16, 35, 100, or 200 sweeps [10] of gauge-invariant Gaussian smearing [11] with a smearing fraction of $\alpha=0.7$, applied at the quark source and sinks in creating the propagators. For the PEVA analyses, this basis is expanded to sixteen operators as described in Sec. II. Before performing the Gaussian smearing, the gauge links to be used are smoothed by applying four sweeps of three-dimensional isotropic stout-link smearing [12] with $\rho=0.1$.

To extract the form factors, we fix the source at time slice $N_{t} / 4=16$. As we use fixed boundary conditions in the time direction, this ensures that the source is sufficiently separated from the boundary to minimize boundary effects. Utilizing the sequential source technique [13], we invert through the current, fixing the current insertion at time slice 21 . We choose time slice 21 by inspecting the projected two-point correlation functions associated with each state and observing that excited-state contaminations are suppressed by time slice 21 . This is evaluated by fitting the effective mass in this region to a single state ansatz verifying that the full covariance $\chi^{2} /$ dof is satisfactory. Choosing the current insertion time in this way allows us to use knowledge of the time slice where the nucleon dominates the two point function to insert the current with the expectation that excited-state systematic errors are contained within the statistical uncertainties. As the correlation matrix is formed in the PEVA approach, opposite-parity contaminations are suppressed at both the source and the sink.

While this technique is a useful guide to choosing a current insertion time, it does not guarantee the elimination of excited state effects. Indeed, as our results will show, excited-state effects can be much worse in three-point functions than two-point functions.

In choosing the variational parameters $t_{0}$ and $\Delta t$ (as defined in Ref. [1]), we have implemented the criteria described in Ref. [14] and compared it with other choices of the variational parameters. In the baryon sector one is always facing challenges with the rapid onset of statistical uncertainties. Moreover, as explored in Ref. [10], the condition number of the correlation matrices deteriorates as one progresses in Euclidean time. Through a careful investigation we found that commencing the variational analysis one slice after the source provides significantly smaller uncertainties in the projected correlators while still providing excellent plateau behavior in the effective mass or energy provided the second time is two or three time slices later. Thus for this work we chose $t_{0}=17$ and $\Delta t=2$.

We then extract the form factors as outlined in Sec. III for every possible sink time and once again look for a plateau consistent with a single-state ansatz.

Performing the sequential source technique through the current requires us to choose our current operators and momentum transfers at inversion time. However, this allows us to vary the sink momentum, and by extension the source momentum, as well as varying the form of the interpolation functions at the sink. This gives us access to several states, as well as a range of values of

$$
Q^{2}=\boldsymbol{q}^{2}-\left(E_{\alpha}\left(\boldsymbol{p}^{\prime}\right)-E_{\alpha}(\boldsymbol{p})\right)^{2} .
$$

In particular, values of $Q^{2}$ well below that encountered in the frames with $\boldsymbol{p}$, or $\boldsymbol{p}^{\prime}=(0,0,0)$ are accessed via kinematics such as $\boldsymbol{p}=\left|\boldsymbol{q}_{\min }\right|(1,0,0), \boldsymbol{p}^{\prime}=\left|\boldsymbol{q}_{\min }\right|(2,0,0)$. The main alternative approach to accessing $Q^{2}$ values in this region is to use twisted boundary conditions to change the momentum discretization, allowing the valence quarks to take different momentum values from the sea quarks. In our approach, all momenta attained by the valence quarks are present in the sea, and thus we avoid the complexities of partial quenching effects inherent in the twisted boundary approach. Table II summarizes the kinematics considered herein.

To begin, we inspect the Euclidean time dependence of $G_{E}\left(Q^{2}\right)$, extracted as outlined in Sec. III. We consider independently the connected contributions to $G_{E}\left(Q^{2}\right)$ from single valence quarks of unit charge. The two flavors considered are the doubly represented quark flavor, or the up quark in the proton $\left(u_{p}\right)$; and the singly represented quark flavor, or the down quark in the proton $\left(d_{p}\right)$.

In the case of perfect optimized operators, there should be no Euclidean time dependence, and the extracted form factors should be perfectly constant (up to statistical fluctuations) after the current insertion. However, in practice a finite operator basis is insufficient to perfectly isolate each state, leading to residual excited-state contaminations. These show up as enhanced or suppressed form factors at 
TABLE II. Different kinematics used in our analysis to access a range of $Q^{2}$ values. The $Q^{2}$ value listed is for the ground-state nucleon at the lightest pion mass of $m_{\pi}=156 \mathrm{MeV}$. The statistical error listed for $Q^{2}$ comes from both the determination of the mass of the state and the conversion to physical units.

\begin{tabular}{lccc}
\hline \hline $\begin{array}{l}\text { Source } \\
\text { momentum } \\
\boldsymbol{p} /\left|\boldsymbol{q}_{\min }\right|\end{array}$ & $\begin{array}{c}\text { Sink } \\
\text { momentum } \\
\boldsymbol{p}^{\prime} /\left|\boldsymbol{q}_{\min }\right|\end{array}$ & $\begin{array}{c}\text { Momentum } \\
\text { transfer } \\
\boldsymbol{q} /\left|\boldsymbol{q}_{\min }\right|\end{array}$ & $Q^{2} / \mathrm{GeV}^{2}$ \\
\hline$(2,0,0)$ & $(3,0,0)$ & $(1,0,0)$ & $0.0833(27)$ \\
$(2,0,1)$ & $(3,0,1)$ & $(1,0,0)$ & $0.0902(28)$ \\
$(1,0,0)$ & $(2,0,0)$ & $(1,0,0)$ & $0.1248(37)$ \\
$(1,0,1)$ & $(2,0,1)$ & $(1,0,0)$ & $0.1301(38)$ \\
$(0,0,0)$ & $(1,0,0)$ & $(1,0,0)$ & $0.1655(48)$ \\
$(0,0,1)$ & $(1,0,1)$ & $(1,0,0)$ & $0.1665(48)$ \\
$(2,0,0)$ & $(3,1,0)$ & $(1,1,0)$ & $0.2211(66)$ \\
$(1,0,0)$ & $(2,1,0)$ & $(1,1,0)$ & $0.2647(78)$ \\
$(0,0,0)$ & $(1,1,0)$ & $(1,1,0)$ & $0.3191(92)$ \\
$(0,-1,0)$ & $(1,0,0)$ & $(1,1,0)$ & $0.3449(100)$ \\
$(1,0,0)$ & $(3,0,0)$ & $(2,0,0)$ & $0.4228(131)$ \\
$(0,0,0)$ & $(2,0,0)$ & $(2,0,0)$ & $0.5989(174)$ \\
$(-1,0,0)$ & $(1,0,0)$ & $(2,0,0)$ & $0.6898(199)$ \\
\hline \hline
\end{tabular}

early Euclidean times. In light of this, care must be taken to select a Euclidean time region in which these excited-state contaminations are suppressed and the single state ansatz is satisfied. To ensure this ansatz is satisfied, we inspect the full covariance $\chi^{2} /$ dof of a constant fit in the proposed plateau region, and require that it lies in an acceptable range $\lesssim 1.2$. At the same time, we ensure that we do not fit excessively noisy points in the tail of the correlator, as they can serve to suppress the $\chi^{2} /$ dof and hide the effects of excited state contamination. In doing so, we sometimes find that the $\chi^{2} /$ dof for a given plateau region differs significantly between the two analyses, and this can lead to selected plateaus that start on different time slices.

In Figs. 1 and 2 we plot both PEVA and conventional extractions of $G_{E}\left(Q^{2}\right)$ with respect to Euclidean sink time at the lightest pion mass of $m_{\pi}=156 \mathrm{MeV}$ and the lowest-momentum kinematics of $\boldsymbol{p}=(0,0,0)$ and $\boldsymbol{p}^{\prime}=$ $\left|\boldsymbol{q}_{\min }\right|(1,0,0)$. We see that starting from time slice 22 , which is immediately after the source, both extractions of $G_{E}\left(Q^{2}\right)$ are quite flat across all time slices considered. However, the errors on $G_{E}\left(Q^{2}\right)$ are sufficiently small to identify a small Euclidean time dependence at early time slices. We find that this dependence is suppressed by time slice 24 and are able to find a clear and clean plateau from 24-27 for both extractions. For both quark flavors considered, there is no significant difference in the fit ranges, extracted values or errors between the two extractions.

The conventional thought is that the opposite-parity contaminations are small. Because they are from heavier states, these contaminations are suppressed by Euclidean time evolution. We will see that this is not the case for the magnetic form factor, where we will present direct evidence for important opposite parity contamination. For the

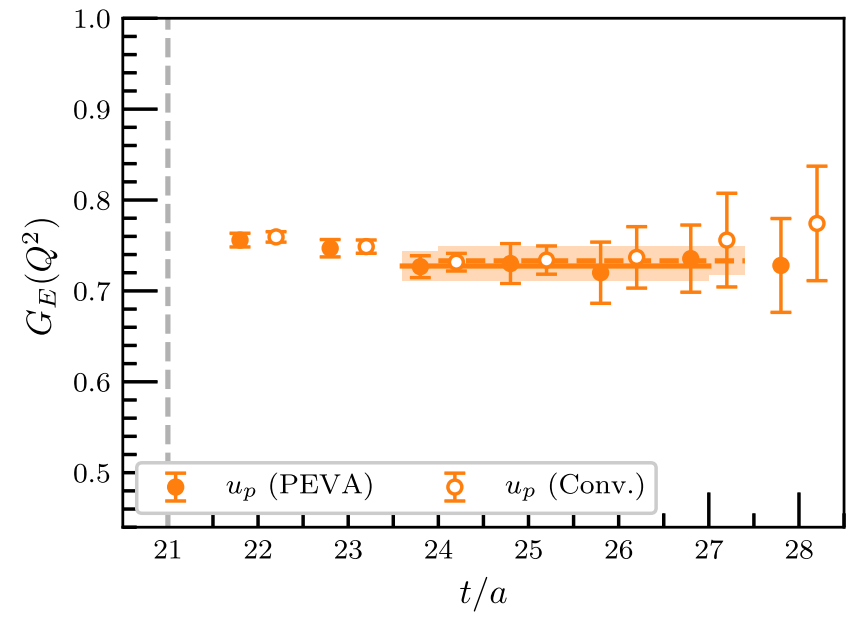

FIG. 1. The contribution of the doubly represented quark flavor to the electric form factor of the ground-state nucleon at $m_{\pi}=156 \mathrm{MeV}$, for the lowest-momentum kinematics, providing $Q^{2}=0.1655(48) \mathrm{GeV}^{2}$. Our fits to the plateaus are illustrated by shaded bands. We plot the conventional analysis with open markers and dashed fit lines and the new PEVA analysis with filled markers and solid fit lines. The source is at time slice 16, and the current is inserted at time slice 21. Both the conventional and PEVA fits are from time slice 24-27.

electric form factor, agreement between the PEVA and conventional analyses can be maintained provided the opposite-parity contaminations contribute to the form factor in a manner similar to that of the parity sector under examination. As a result, despite their continued presence, the opposite-parity contaminations do not significantly perturb the value of the electric form factor.

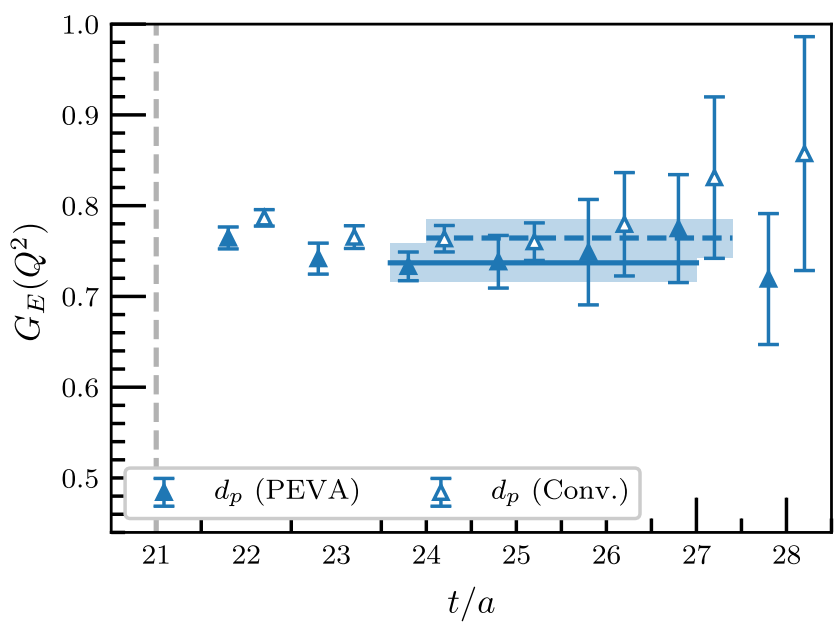

FIG. 2. The contribution of the singly represented quark flavor to the electric form factor of the ground-state nucleon. The conventions used in this plot are the same as used in Fig. 1. The kinematics are also the same, with $m_{\pi}=156 \mathrm{MeV}$, and $Q^{2}=0.1655(48) \mathrm{GeV}^{2}$. Both the conventional and PEVA fits are from time slice $24-27$. 


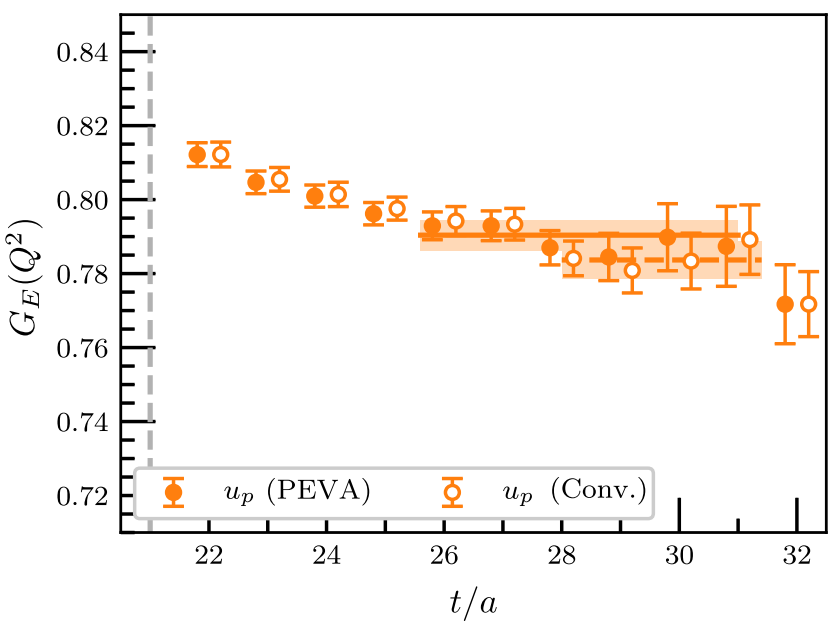

FIG. 3. The contribution of the doubly represented quark flavor to the electric form factor of the ground-state nucleon at $m_{\pi}=570 \mathrm{MeV}$, for the lowest-momentum kinematics, providing $Q^{2}=0.1444(44) \mathrm{GeV}^{2}$. The conventions used in this plot are the same as used in Fig. 1. The PEVA fits start at time slice 26, whereas the conventional fits start at time slice 28 . Note that this plot has been scaled up significantly to make the difference between the two fits visible.

At the heavier pion masses, the statistical noise in the form factor extractions decreases, and the plateau region shifts somewhat. However for all five masses, the qualitative behavior described above remains true, save for the following anomalies. At $m_{\pi}=570 \mathrm{MeV}$, the plateaus from PEVA start two time slices earlier than those from the conventional analysis. For example, Fig. 3 shows the plateaus for the doubly represented quark flavor. This is potentially a signal of opposite-parity contaminations entering into the analysis. However, there is no statistically significant difference in the fit values from the two methods and the different plateaus do not show up at any of the other masses considered, so it is inconclusive.

We can also consider changing the momenta of the initial and final states, both by changing the momentum transfer, and by boosting both the initial and final states without changing the three-momentum transfer.

If we do this for the $m_{\pi}=156 \mathrm{MeV}$ ensemble, where we previously found consistent plateaus between PEVA and a conventional analysis, we find some discrepancies. In Figs. 4 and 5, we boost the initial state momentum to $\boldsymbol{p}=$ $\left|\boldsymbol{q}_{\text {min }}\right|(-1,0,0)$ and increase the momentum transfer to $\boldsymbol{q}=\left|\boldsymbol{q}_{\mathrm{min}}\right|(2,0,0)$, leading to a significant increase in $Q^{2}$. In this case, we find that the PEVA plateaus start one time slice earlier than the conventional plateaus. They have consistent plateau values, but due to the earlier onset of the PEVA plateaus the statistical error is reduced. These results suggest that there are contaminations in the extraction of $G_{E}\left(Q^{2}\right)$ with the conventional analysis at this mass. However the differences are not consistent across all higher-momentum kinematics, and are not enough to

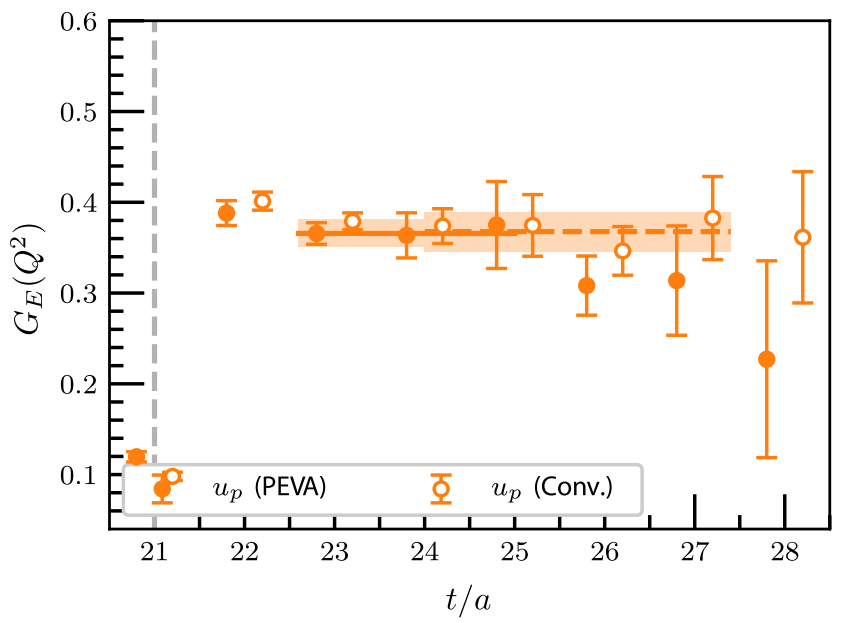

FIG. 4. Contributions of $u_{p}$ to the ground state $G_{E}\left(Q^{2}\right)$ at $m_{\pi}=$ $156 \mathrm{MeV}$ for $\boldsymbol{p}=\left|\boldsymbol{q}_{\min }\right|(-1,0,0)$ and $\boldsymbol{p}^{\prime}=\left|\boldsymbol{q}_{\min }\right|(1,0,0)$, providing $Q^{2}=0.690(20) \mathrm{GeV}^{2}$. The conventions used in this plot are the same as in Fig. 1. The PEVA fit starts from time slice 23, but the conventional analysis starts from time slice 24 .

categorically ascribe these problems to opposite-parity contamination. We do note that when there is a difference in the onset of the plateaus, the PEVA plateau always starts earlier.

For the other four masses, almost all kinematics have identical plateaus in $G_{E}\left(Q^{2}\right)$ from both analyses, save for $m_{\pi}=570 \mathrm{MeV}$, which once again has consistently earlier plateaus for PEVA than the conventional analysis. It is unclear why $m_{\pi}=570 \mathrm{MeV}$ has inconsistent plateaus at a range of kinematics when the other three heavy masses do not. However, it is clear that whatever opposite-parity contaminations are occurring, they are not affecting the $G_{E}\left(Q^{2}\right)$ values extracted, at least within our current statistical uncertainties.

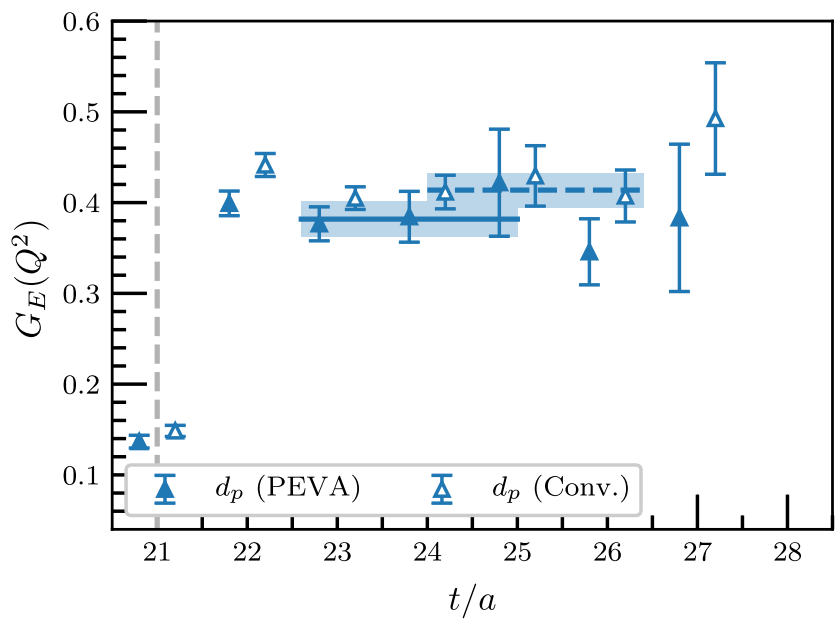

FIG. 5. Contributions of $d_{p}$ to the ground state $G_{E}\left(Q^{2}\right)$. Pion mass and kinematics are as in Fig. 4 above. The conventions used in this plot are the same as in Fig. 1. The PEVA fit starts from time slice 23 , but the conventional analysis starts from time slice 24 . 


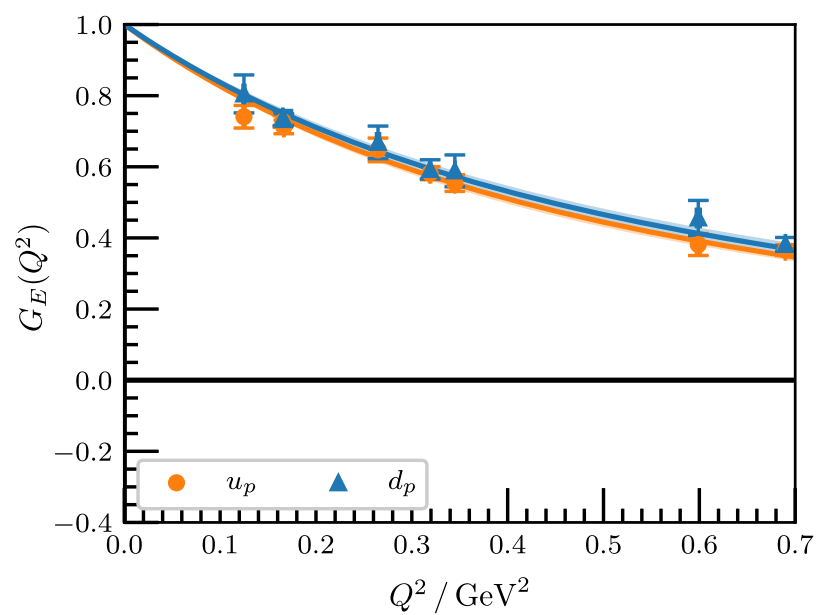

FIG. 6. Contributions from individual quark flavors to the electric form factor of the ground-state nucleon at $m_{\pi}=156 \mathrm{MeV}$. The shaded regions are dipole fits to the form factor, with lines indicating the central values. The $y$-axis intercept is fixed to one, as we are using an improved conserved vector current and the quarks are taken with unit charge. The errors on these fits are small enough that the shaded bands are barely distinguishable from the central lines. The fits correspond to a charge radius of 0.684(19) fm for the doubly represented quark $\left(u_{p}\right)$ and $0.659(21)$ fm for the singly represented quark $\left(d_{p}\right)$.

Across all five masses, we consistently find that at higher momenta there is more statistical noise in the extraction of $G_{E}\left(Q^{2}\right)$.

In Fig. 6, we take the plateau values from each of the kinematics listed in Table II at $m_{\pi}=156 \mathrm{MeV}$ and plot their $Q^{2}$ dependence. We exclude any kinematics for which we are unable to find a clear plateau, or the variational analysis produces a negative generalized eigenvalue (as negative eigenvalues indicate issues with the variational analysis, and can cause problems with state identification). We see the contributions from both quark flavors are very similar and each agrees well with a dipole ansatz

$$
G_{D}\left(Q^{2}\right)=\frac{G_{0}}{\left(1+Q^{2} / \Lambda^{2}\right)^{2}},
$$

with $G_{0}$ fixed to one, as we are working with single quarks of unit charge. These fits correspond to an RMS charge radius of $\left\langle r^{2}\right\rangle^{1 / 2}=\sqrt{12} / \Lambda=0.684(19)$ fm for the doubly represented quark flavor and $0.659(21)$ fm for the singly represented quark flavor. That these values are smaller than the physical expressions can be ascribed to the finite volume of the lattice [15]. For brevity, we omit similar plots for the other four masses.

In order to compute the form factors of the proton, $G_{E p}\left(Q^{2}\right)$, and neutron, $G_{E n}\left(Q^{2}\right)$, we need to take the correct linear combinations of the contributions from the doubly and singly represented quark flavors to reintroduce

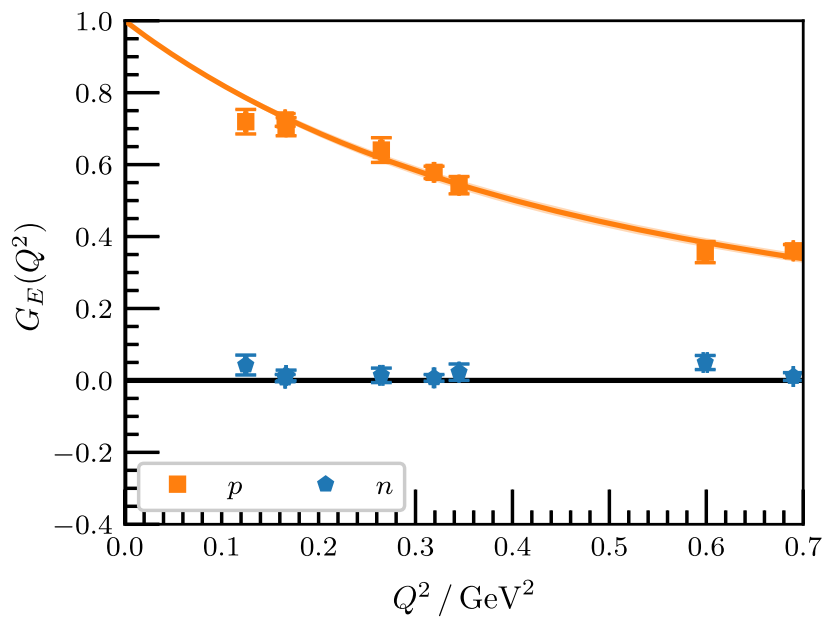

FIG. 7. $G_{E}\left(Q^{2}\right)$ for the ground-state proton and neutron at $m_{\pi}=156 \mathrm{MeV}$. The shaded region corresponds to a dipole fit to the proton form factor, with a charge radius of $0.691(19) \mathrm{fm}$.

the multiplicity of the doubly represented quark and the physical charges of the up and down quarks.

In Fig. 7 and Table III, we present the electric form factors obtained by these combinations for the lightest pion mass considered here.

In this work, we only consider connected contributions to the nucleon form factors. There is no a priori reason that the disconnected loops could not be included in a PEVA calculation. They were simply omitted from the analysis presented here for computational efficiency. The disconnected loop contributions to the proton and neutron should be approximately the same (exactly the same in our lattice calculations, as we are in the isospin symmetric limit). Hence, if we take the isovector combination $G_{E p}\left(Q^{2}\right)-G_{E n}\left(Q^{2}\right)$, the disconnected loop contributions will cancel. The form factor values for this combination are also presented in Table III.

The form factor for the neutrally charged neutron is close to zero for all masses considered, as expected. Similar to the linear combinations taken for the form factors, we can

TABLE III. $G_{E}\left(Q^{2}\right)$ at $m_{\pi}=156 \mathrm{MeV}$ for all acceptable kinematics. We present results for the ground-state proton and neutron, as well as isovector combination $G_{E p}\left(Q^{2}\right)-G_{E n}\left(Q^{2}\right)$ (which is insensitive to disconnected loop corrections).

\begin{tabular}{lllc}
\hline \hline$Q^{2} / \mathrm{GeV}^{2}$ & $G_{E p}\left(Q^{2}\right)$ & $G_{E n}\left(Q^{2}\right)$ & $G_{E p}\left(Q^{2}\right)-G_{E n}\left(Q^{2}\right)$ \\
\hline $0.1248(37)$ & $0.719(34)$ & $0.043(28)$ & $0.677(51)$ \\
$0.1655(48)$ & $0.724(18)$ & $0.006(10)$ & $0.718(23)$ \\
$0.1665(48)$ & $0.705(25)$ & $0.014(15)$ & $0.691(38)$ \\
$0.2647(78)$ & $0.640(34)$ & $0.014(20)$ & $0.626(44)$ \\
$0.3191(92)$ & $0.578(17)$ & $0.007(9)$ & $0.571(18)$ \\
$0.3449(100)$ & $0.543(24)$ & $0.023(23)$ & $0.520(38)$ \\
$0.5989(174)$ & $0.357(29)$ & $0.050(20)$ & $0.307(35)$ \\
$0.6898(199)$ & $0.360(17)$ & $0.011(11)$ & $0.350(25)$ \\
\hline \hline
\end{tabular}


combine the squared charge radii from the individual quark sectors with the appropriate multiplicities and charge factors to obtain the squared charge radius of the neutron. For all five pion masses, we find a small negative value. For example, at $m_{\pi}=156 \mathrm{MeV}$, the neutron's squared charge radius is $-0.022(9) \mathrm{fm}$. This is qualitatively consistent with the negative squared charge radii observed in experiment. A more quantitative discussion of this effect requires knowledge of the disconnected loop contributions, which are not considered in this work.

The form factor of the proton matches well with a dipole fit with $G_{0}$ fixed to one (the charge of the proton). As expected, the charge radii extracted from these dipole fits approach the experimentally measured proton charge radius from below as the pion mass is reduced towards the physical point.

As discussed in Refs. [16,17], the exact physical value of the proton radius has been a puzzle for the last seven years, since precision laser spectroscopy of muonic hydrogen yielded a proton radius of $0.84087(39) \mathrm{fm}$ [18] in 2010. This value is $4.6 \%$, or $5.6 \sigma$ lower than the CODATA 2014 world average of 0.8751(61) fm [19], from a combination of laser spectroscopy of electronic hydrogen and deuterium, and elastic electron scattering. Recent precision results from new laser spectroscopy of electronic hydrogen provide a proton radius of $0.8335(95) \mathrm{fm}$ [20], which agrees with the muonic hydrogen radius. This suggests that the discrepancy is likely due to systematic errors in the existing results for electronic hydrogen and elastic electron scattering.

Returning to our results, in Fig. 8, we plot the charge radii obtained from dipole fits to the isovector combination as a function of the squared pion mass. We see that the pion-mass dependence is quite smooth, suggesting that the structure of the state is fairly consistent at all five masses

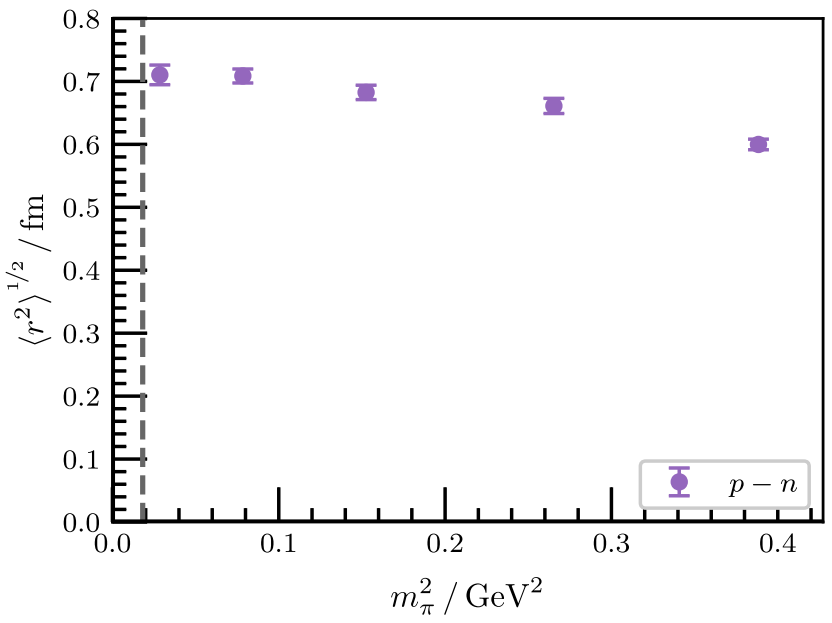

FIG. 8. Quark-mass dependence of charge radii from dipole fits to the isovector combination $G_{E p}\left(Q^{2}\right)-G_{E n}\left(Q^{2}\right)$. We see a clear trend to larger radii as the pion mass approaches the physical point, represented by the dashed vertical line. considered here. It has a clear trend of increasing charge radius as the mass is reduced. This effect is in accord with the expectations of finite-volume chiral perturbation theory [15].

For all pion masses and kinematics considered in this paper, in the specific case of the electric form factor, there is no conclusive evidence of opposite parity contaminations. Both the PEVA and conventional variational analysis show clear and clean plateaus in $G_{E}\left(Q^{2}\right)$ with good excited state control. This supports previous work demonstrating the utility of variational analysis techniques in calculating baryon matrix elements [21,22]. By using such techniques we are able to cleanly isolate precise values for the Sachs electric form factor of the ground-state proton and neutron.

\section{SACHS MAGNETIC FORM FACTOR}

Moving on to $G_{M}\left(Q^{2}\right)$, we once again begin with the lightest pion mass and the lowest momenta. Here, we present results in terms of nuclear magnetons, $\mu_{N} \equiv \frac{e \hbar}{2 m_{p}^{\text {Phys }}}$, defined in terms of the physical proton mass, $m_{p}^{\text {Phys }}$. In Fig. 9, we see that while the signal is noisier than $G_{E}\left(Q^{2}\right)$, the excited-state contaminations present at early Euclidean times are less significant, and for both the PEVA and conventional analyses we are able to find a plateau from time slice 23 to 25 . We are cautious in fitting noisy data and restrict fit regimes to avoid large fluctuations. Figure 10

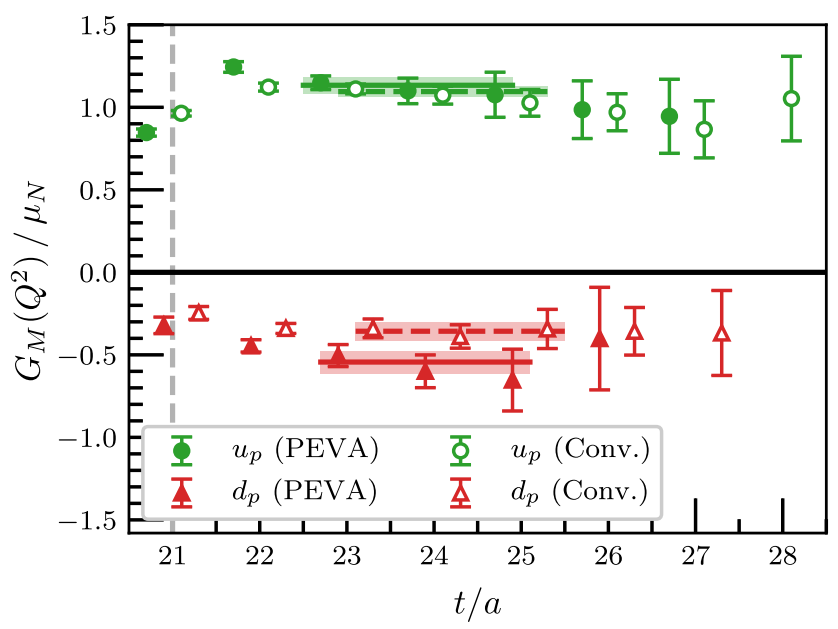

FIG. 9. The contributions to the magnetic form factor from single quarks of unit charge for the ground-state nucleon at $m_{\pi}=$ $156 \mathrm{MeV}$ for the lowest-momentum kinematics, providing $Q^{2}=0.1655(48) \mathrm{GeV}^{2}$. We plot the conventional analysis with open markers and the new PEVA analysis with filled markers. Our fits to the plateaus are illustrated by shaded bands, with the central value indicated by dashed lines for the conventional analysis, and solid lines for the PEVA analysis. The plateau regions for both analyses are consistent, starting from time slice 23 for all four fits, but the value of the conventional plateau for the singly represented quark $\left(d_{p}\right)$ has a magnitude approximately $35 \%$ lower than the PEVA plateau. 


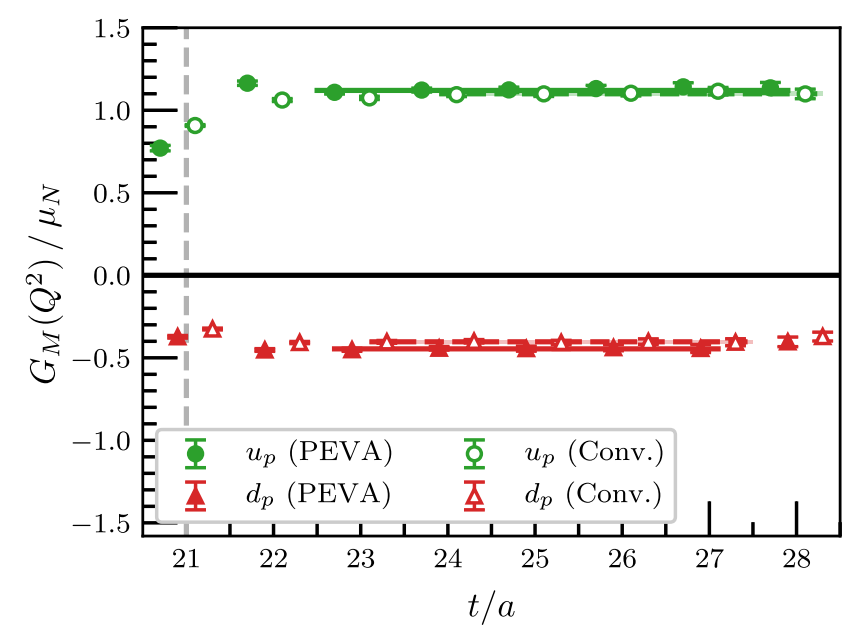

FIG. 10. The contributions to the magnetic form factor from single quarks of unit charge for the ground-state nucleon at $m_{\pi}=$ $570 \mathrm{MeV}$ for the lowest-momentum kinematics, providing $Q^{2}=0.1444(44) \mathrm{GeV}^{2}$. The plateau regions for both analyses are consistent, starting from time slice 23 for all four fits, but the value of the conventional plateau for the singly represented quark $\left(d_{p}\right)$ has a magnitude slightly lower than the PEVA plateau.

illustrates a similar plot for $m_{\pi}=570 \mathrm{MeV}$. Here the extended plateau is from time slice 23 to 27 and is more representative of the three heaviest pion masses considered.

Contrary to the electric case, there is a statistically significant difference in the values of the plateaus from the PEVA and conventional analysis for the singly represented quark flavor. If we take the correlated ratio of $G_{M}\left(Q^{2}\right)$ from the conventional analysis to $G_{M}\left(Q^{2}\right)$ from the PEVA analysis, we get a value of $0.66(9)$. This ratio is clearly less than 1 , indicating that the magnitude of the form factor is being significantly underestimated in the conventional analysis. This suggests that despite finding a plateau, the conventional analysis is being affected by opposite-parity contaminations that are introducing a systematic error of approximately $35 \%$.

This is the strongest effect we see across all kinematics considered. However, the conventional plateaus for other kinematics still show a statistically significant deviation from the PEVA plateaus despite having the same fit regions and acceptable $\chi^{2}$ values. In Fig. 11, we plot the correlated ratio discussed above for the kinematics that give the least noisy extractions of $G_{M}\left(Q^{2}\right)$ with acceptable plateaus and positive generalized eigenvalues. We see that for the doubly represented quark sector, while some kinematics are consistent with unity, others sit more than one standard deviation low. The full covariance $\chi^{2} /$ dof for an ansatz of unity across all kinematics for which there are acceptable plateaus (including ratios not on this graph) is 5.0. This indicates a significant disagreement between the two analyses, suggesting that the conventional variational analysis is likely contaminated by opposite-parity states. While it is not clear that the effect will be the same across

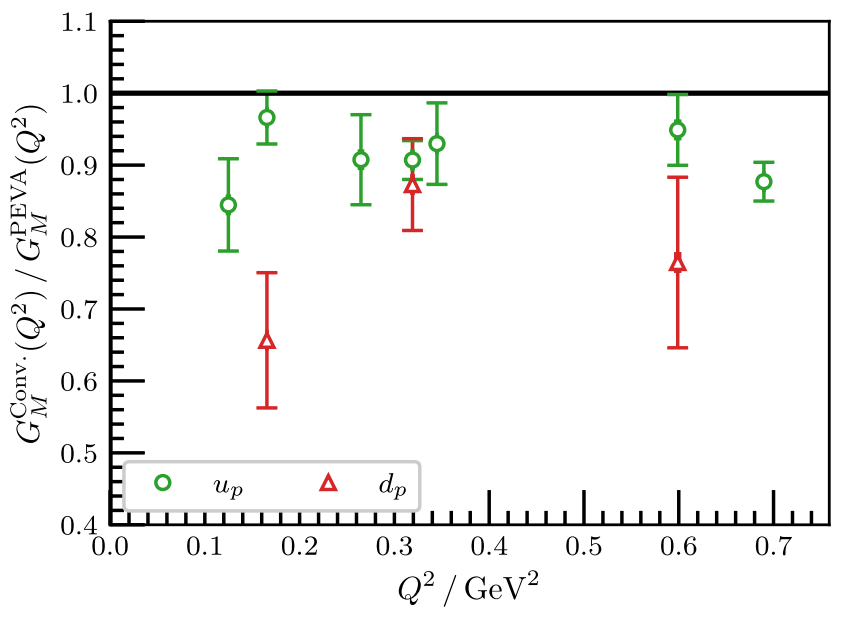

FIG. 11. Ratios of conventional plateaus to PEVA plateaus for ground state $G_{M}\left(Q^{2}\right)$ at $m_{\pi}=156 \mathrm{MeV}$. For clarity, ratios with large statistical errors have been excluded from the plot. If the plateaus were consistent, the points should be distributed about 1.0. For the doubly represented quark flavor $\left(u_{p}\right)$, some kinematics match this expectation, but others sit more than one standard deviation low. The singly represented quark flavor $\left(d_{p}\right)$ appears to show an even larger effect, with the ratios shifting even further away from unity albeit with larger statistical errors.

all kinematics, we can take a correlated weighted average across all of the kinematics with valid plateaus to obtain an estimate for the size of the effect. Doing so, we obtain a value of $0.92(2)$, indicating that this quark flavor sees errors of 5\%-10\%. Removing the noisiest points as in Fig. 11 does not significantly alter these results, giving a $\chi^{2} /$ dof of 5.4 and a weighted average of $0.91(2)$.

The singly represented quark flavor $\left(d_{p}\right)$ potentially shows an even larger effect, with a weighted average of $0.83(5)$. The $\chi^{2} /$ dof is lower due to larger statistical errors, taking a value of 2.9. However, it is still quite large, indicating the difference between the two analyses is significant. While removing the noisiest points as in Fig. 11 does increase the $\chi^{2} /$ dof to 6.4 , it does not significantly change the weighted average, giving 0.78(5). These results indicate the presence of opposite-parity contaminations, which introduce systematic errors of $10 \%-20 \%$ for $d_{p}$, and perhaps more for some specific kinematics.

As the states become less relativistic at larger quark masses, we see a reduction in the amount of parity mixing that occurs, and consequentially in the size of the systematic errors, particularly at the heaviest two masses. However, we still observe statistically significant deviations of the ratio below unity. For the heaviest two masses of $570 \mathrm{MeV}$ and $702 \mathrm{MeV}$, we see a systematic underestimation of the singly represented quark contributions by $5 \%-10 \%$ and at the remaining masses of $411 \mathrm{MeV}$ and $296 \mathrm{MeV}$, we see a 10\%-15\% underestimation.

These results provide strong evidence for opposite-parity contaminations in conventional extractions. These contaminations have a clear effect on the extracted magnetic form 


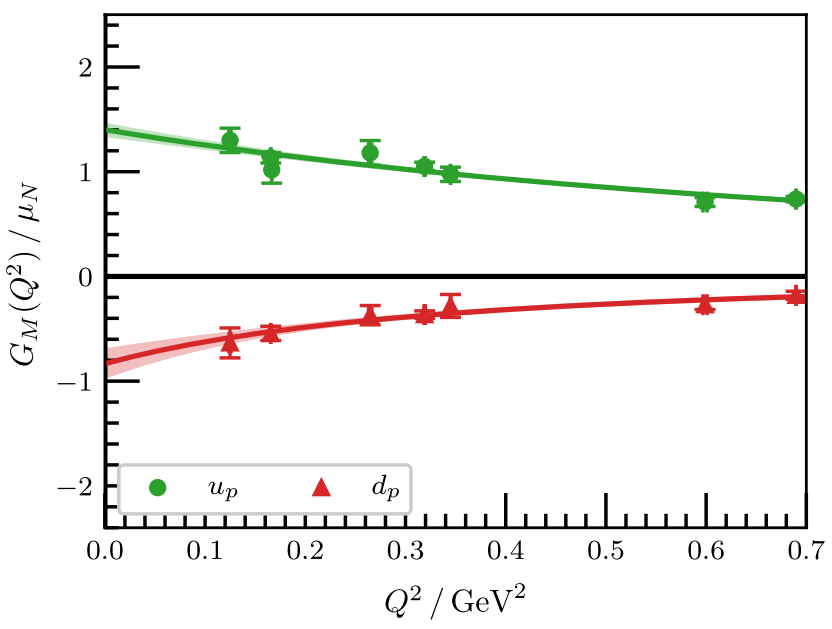

FIG. 12. Quark-flavor contributions to ground state $G_{M}\left(Q^{2}\right)$ at $m_{\pi}=156 \mathrm{MeV}$. The shaded regions are dipole fits to the form factor, corresponding to magnetic radii of 0.514(30) fm for the doubly represented quark flavor $\left(u_{p}\right)$ and $0.85(11) \mathrm{fm}$ for the singly represented quark flavor $\left(d_{p}\right)$.

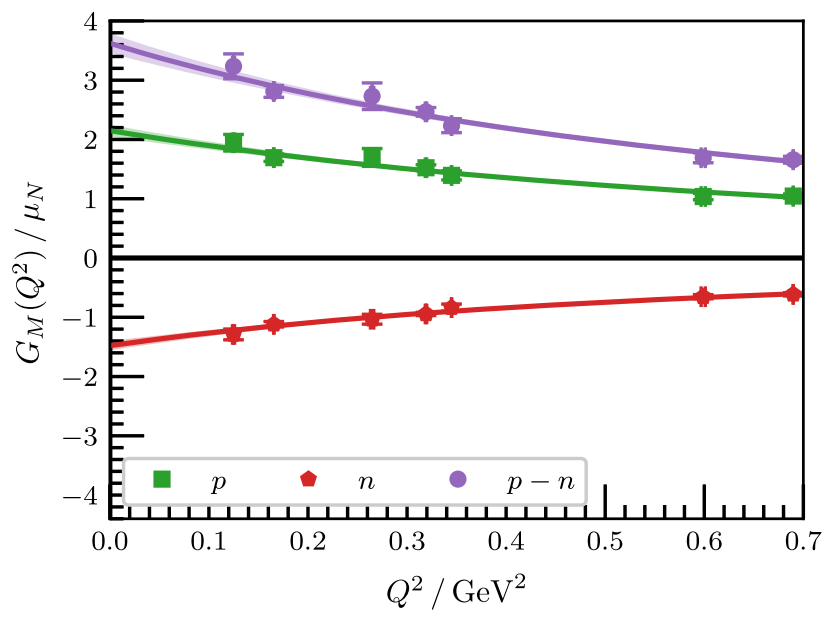

FIG. 13. $G_{M}\left(Q^{2}\right)$ for the ground-state proton and neutron at $m_{\pi}=156 \mathrm{MeV}$. The shaded region corresponds to a dipole fit to the form factor, with a magnetic radius of 0.551(29) fm for the proton and 0.618(31) fm for the neutron. We also include the isovector combination $\left(G_{M p}\left(Q^{2}\right)-G_{M n}\left(Q^{2}\right)\right)$, which is insensitive to disconnected loop corrections. factor at all five pion masses, on the order of $10 \%$ for the doubly represented quark sector $\left(u_{p}\right)$ and $20 \%$ for the singly represented quark sector $\left(d_{p}\right)$ at the lighter masses. Moving forward, use of the PEVA technique will be critical in precision calculations of $G_{M}\left(Q^{2}\right)$ for the ground-state nucleon, for which such systematic errors are unacceptable.

We now proceed to examine the extracted form factors. In light of the opposite-parity contaminations present in the conventional extractions, we focus only on the PEVA results. Figure 12 shows the $Q^{2}$ dependence of the contribution to $G_{M}\left(Q^{2}\right)$ from each quark flavor at $m_{\pi}=156 \mathrm{MeV}$. We see good agreement with a dipole ansatz, with magnetic radii of 0.514(30) fm for the doubly represented quark flavor and $0.85(11)$ fm for the singly represented quark flavor.

Similar to the electric form factor case described in Sec. IV, we take linear combinations of the contributions from the doubly and singly represented quark flavors to obtain the magnetic form factors of the proton $\left(G_{M p}\left(Q^{2}\right)\right)$ and neutron $G_{M n}\left(Q^{2}\right)$. In addition, we can take the isovector combination $\left(G_{M p}\left(Q^{2}\right)-G_{M n}\left(Q^{2}\right)\right)$ to cancel out disconnected loop contributions.

We plot these combinations for the lightest pion mass in Fig. 13, and present the values in Table IV. At all five masses, the magnetic form factors of both the proton and the neutron agree well with a dipole fit. The magnetic radius obtained from each of these fits is close to the electric charge radius of the proton extracted from $G_{E}\left(Q^{2}\right)$ at the same pion mass.

In Fig. 14, we plot the quark-mass dependence of charge radii obtained from the dipole fits to the isovector combination $G_{M p}\left(Q^{2}\right)-G_{M n}\left(Q^{2}\right)$. We can once again see a quark-mass dependence, with increasing charge radius at lighter pion masses, aside from the lightest mass, where the fit is getting too noisy to clearly distinguish a trend. At the same time, $G_{M}(0)$ is increasing. This is in agreement with expectations from chiral perturbation theory $[23,24]$. $G_{M}(0)$ corresponds to the magnetic moment, which will be studied in more detail in the next section.

In this section, we demonstrated the importance of the PEVA technique in controlling systematic errors arising from opposite-parity contaminations in extractions of the

TABLE IV. $G_{M}\left(Q^{2}\right)$ at $m_{\pi}=156 \mathrm{MeV}$ for all acceptable kinematics. We present results for the ground-state proton and neutron, as well as isovector combination $G_{M p}\left(Q^{2}\right)-G_{M n}\left(Q^{2}\right)$ (which should be free from disconnected loop corrections).

\begin{tabular}{lccc}
\hline \hline$Q^{2} / \mathrm{GeV}^{2}$ & $G_{M p}\left(Q^{2}\right) / \mu_{N}$ & $G_{M n}\left(Q^{2}\right) / \mu_{N}$ & $\left(G_{M p}\left(Q^{2}\right)-G_{M n}\left(Q^{2}\right)\right) / \mu_{N}$ \\
\hline $0.1248(37)$ & $1.94(14)$ & $-1.29(9)$ & $3.23(21)$ \\
$0.1655(48)$ & $1.69(6)$ & $-1.12(5)$ & $2.81(10)$ \\
$0.2647(78)$ & $1.70(15)$ & $-1.03(8)$ & $2.73(22)$ \\
$0.3191(92)$ & $1.52(5)$ & $-0.94(3)$ & $2.47(7)$ \\
$0.3449(100)$ & $1.39(8)$ & $-0.84(6)$ & $2.23(12)$ \\
$0.5989(174)$ & $1.04(5)$ & $-0.65(4)$ & $1.69(8)$ \\
$0.6898(199)$ & $1.05(3)$ & $-0.61(3)$ & $1.66(6)$ \\
\hline \hline
\end{tabular}




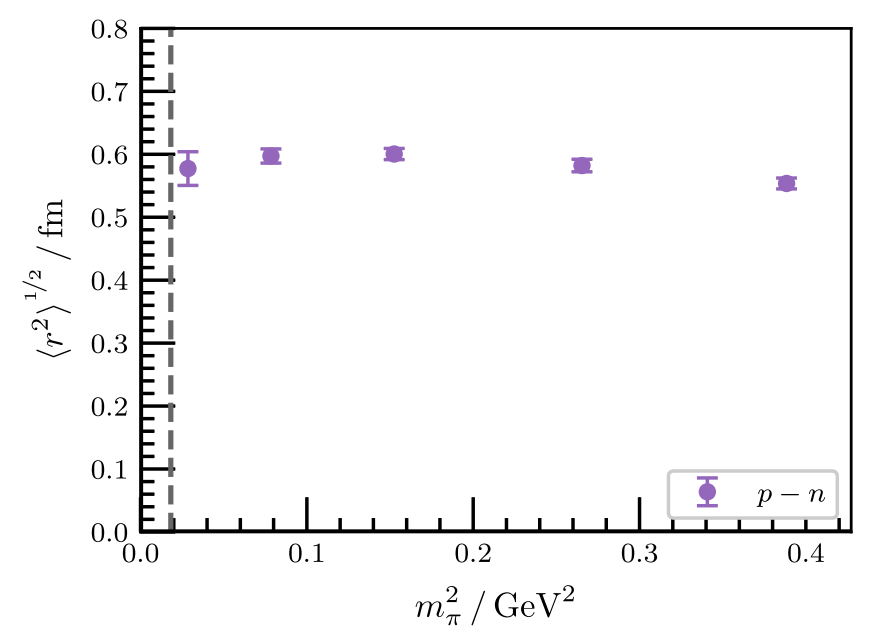

FIG. 14. Quark-mass dependence of charge radii obtained from dipole fits to the isovector magnetic moment $\left(G_{M p}\left(Q^{2}\right)-G_{M n}\left(Q^{2}\right)\right)$. As in Fig. 8, the dashed line corresponds to the physical pion mass.

magnetic form factor for the ground-state nucleon. Due to these opposite-parity contaminations, the conventional analysis underestimates the contribution to the magnetic form factor from the singly represented quark sector by $\sim 20 \%$ at light pion masses, whereas the PEVA technique removes the contaminations and gives improved results.

\section{MAGNETIC DIPOLE MOMENT}

Returning to the individual quark flavor contributions, and noting that the observed $Q^{2}$ dependence of $G_{E}\left(Q^{2}\right)$ and $G_{M}\left(Q^{2}\right)$ is very similar, we hypothesize that $G_{M}\left(Q^{2}\right)$ and $G_{E}\left(Q^{2}\right)$ have the same scaling in $Q^{2}$ over the range considered here. If this hypothesis is valid, then the ratio of $G_{M}\left(Q^{2}\right)$ to $G_{E}\left(Q^{2}\right)$ should be independent of $Q^{2}$. Since we are working with an improved conserved vector current, and single quarks of unit charge, $G_{E}(0)=1$ exactly, and $G_{M}(0)$ is the contribution of the quark flavor to the magnetic moment (up to scaling by the physical charge). Hence, the ratio

$$
\mu_{\mathrm{Eff}}\left(Q^{2}\right) \equiv \frac{G_{M}\left(Q^{2}\right)}{G_{E}\left(Q^{2}\right)}
$$

is expected to be constant in $Q^{2}$, and equal to the contribution to the magnetic moment from the given quark flavor.

Experimental results show that at high $Q^{2}$, $\mu G_{E}\left(Q^{2}\right) / G_{M}\left(Q^{2}\right)$ diverges significantly from unity [25], so our hypothesis must break down at sufficiently high $Q^{2}$. However, over the low $Q^{2}$ range we consider here, these experimental results show that $\mu G_{E}\left(Q^{2}\right) / G_{M}\left(Q^{2}\right)$ is close to one, and hence within this range $G_{M}\left(Q^{2}\right) / G_{E}\left(Q^{2}\right)$ approximates the magnetic moment.

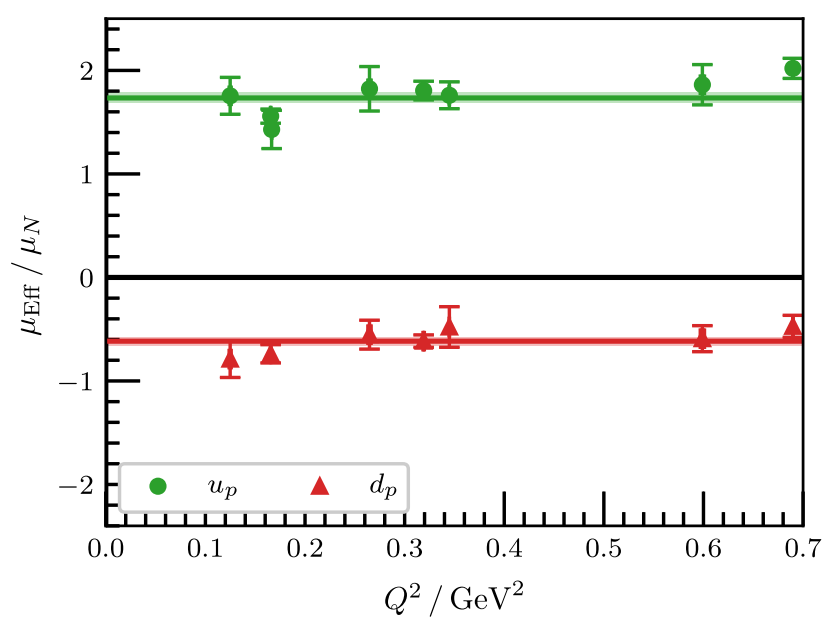

FIG. 15. $\mu_{\mathrm{Eff}}\left(Q^{2}\right)$ for individual quark flavors in the ground state nucleon at $m_{\pi}=156 \mathrm{MeV}$. The narrow shaded bands are constant fits to the effective magnetic moment. They correspond to magnetic moment contributions of 1.734(56) $\mu_{N}$ for the doubly represented quark and $-0.616(44) \mu_{N}$ for the singly represented quark.

For all five pion masses, we find that $\mu_{\mathrm{Eff}}\left(Q^{2}\right)$ is indeed approximately constant across the $Q^{2}$ range considered. For example, Fig. 15 shows the $Q^{2}$ dependence of $\mu_{\mathrm{Eff}}\left(Q^{2}\right)$ at the lightest pion mass. The remaining masses show very similar $Q^{2}$ dependence. By taking a constant fit across all kinematics we obtain a estimate for the contributions to the magnetic moment of the nucleon from single quarks of unit charge. In the graphs shown here, the statistical errors on this fit are small, and the shaded band showing these errors is almost indistinguishable from the solid line indicating the central value of the fit. Figure 16 shows the pion mass dependence of these fits. These individual quark-flavor

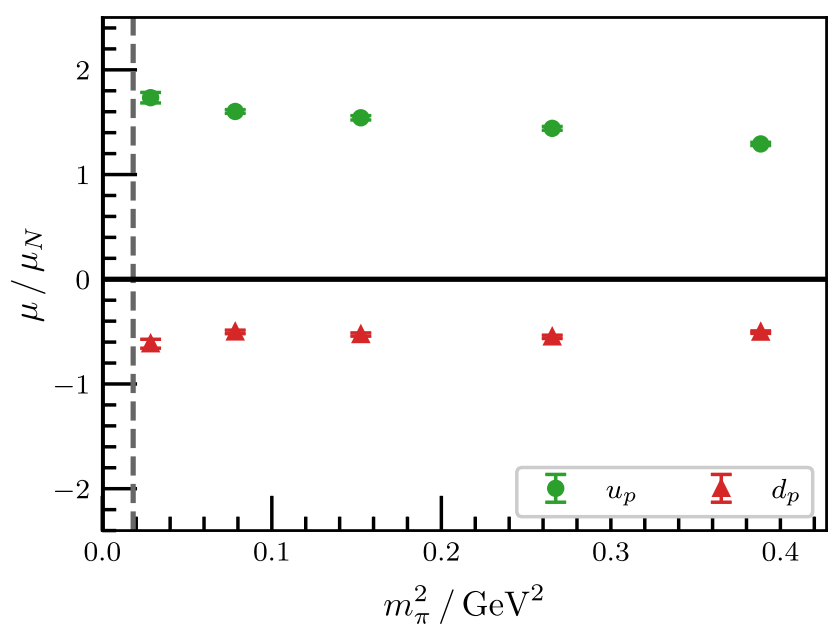

FIG. 16. Pion-mass dependence of contributions to the groundstate magnetic moment from the doubly represented quark sector $\left(u_{p}\right)$ and the singly represented quark sector $\left(d_{p}\right)$. The vertical dashed line shows the physical pion mass. 


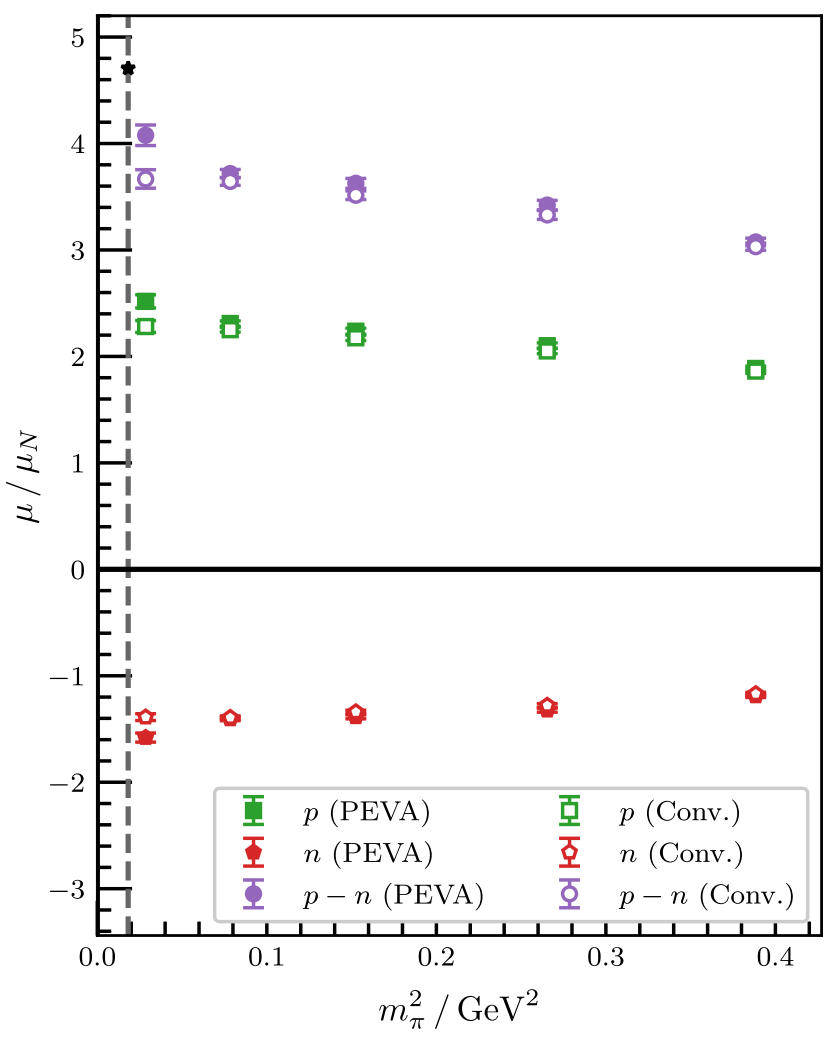

FIG. 17. Pion-mass dependence of the extracted magnetic moment for the ground-state proton and neutron. To cancel out any disconnected loop contributions, we plot the isovector combination $\mu_{p}-\mu_{n}$. As the physical point is approached, the trend in this combination approaches but does not quite reach the physical value of $4.70 \mu_{N}$ [19], represented by a black star.

contributions show a smooth pion-mass dependence with an enhancement of the magnetic moments at low pion mass consistent with chiral perturbation theory $[23,24,26]$.

We can take the linear combinations discussed in Sec. IV to obtain the magnetic moments of the ground-state proton and neutron. The quark mass dependence of these combinations is illustrated in Fig. 17, as well as in Table V.

TABLE V. Magnetic moments at $m_{\pi}=156 \mathrm{MeV}$ for all five pion masses. We present results for the ground-state proton and neutron, for both the PEVA analysis and the conventional parity projected analysis. We see that the conventional analysis consistently underestimates the magnetic moments, with the largest effect at smaller pion masses, where it reaches approximately $10 \%$.

\begin{tabular}{lcccc}
\hline \hline & $\begin{array}{c}\mu_{p} / \mu_{N} \\
m_{\pi}^{2} / \mathrm{GeV}^{2}\end{array}$ & $\begin{array}{c}\mu_{p} / \mu_{N} \\
\text { (CEVA) }\end{array}$ & $\begin{array}{c}\mu_{n} / \mu_{N} \\
\text { (Conv.) }\end{array}$ & $\begin{array}{c}\mu_{n} / \mu_{N} \\
\text { (CoVA) }\end{array}$ \\
\hline $0.3884(113)$ & $1.89(2)$ & $1.86(2)$ & $-1.19(1)$ & $-1.17(1)$ \\
$0.2654(81)$ & $2.10(3)$ & $2.05(3)$ & $-1.32(2)$ & $-1.28(2)$ \\
$0.1525(43)$ & $2.24(3)$ & $2.17(3)$ & $-1.39(2)$ & $-1.34(2)$ \\
$0.0784(25)$ & $2.31(2)$ & $2.25(2)$ & $-1.41(2)$ & $-1.39(2)$ \\
$0.0285(12)$ & $2.52(6)$ & $2.28(6)$ & $-1.58(4)$ & $-1.39(3)$ \\
\hline \hline
\end{tabular}

We also present the equivalent magnetic moment extractions from the conventional analysis. At the heavier pion masses, the conventional analysis slightly underestimates the magnetic moment values. At the lighter pion masses, this discrepancy grows rapidly, reaching approximately $10 \%$ at the lightest pion mass considered here.

It is of interest to understand the origin of the difference between the PEVA and conventional analyses at the lightest pion mass considered here. Inspecting the excited state spectrum and the structure of the optimized operators at the lightest two masses shows some difference, but no clear indication of why the opposite-parity contaminations would be so much stronger at the lightest mass. However, a detailed investigation of the negative parity spectrum gives a hint at a possible cause. At the heavier pion masses, the localized negative parity excitations have magnetic moments similar to quark model expectations for the $N^{*}(1535)$ and $N^{*}(1650)$ resonances, as presented in Ref. [27]. However, at the lighter pion masses, the magnetic moments shift away from the quark model expectations, suggesting an increasing role for multiparticle states in the negative-parity spectrum. This leads to a change in the nature of the localized negative-parity states that couple well to the localized operators used in this work, which in turn can significantly alter the effects of opposite-parity contaminations on the ground state matrix elements.

The magnetic moments of the proton and neutron extracted by PEVA have a similar quark mass dependence to the individual quark-flavor contributions and are close to the experimental values of 2.7928473508(85) $\mu_{N}$ for the proton, and $-1.91304273(45) \mu_{N}$ for the neutron [19]. The small discrepancy between our results and the physical values is due to a combination of disconnected loop contributions which are not included in our calculation, and finite-volume effects. To avoid the disconnected loop corrections, we compare the isovector combination $\mu_{p}-\mu_{n}$ to the equivalent combination of the experimentally determined magnetic moments. Doing this, we find that we underestimate the experimental value by around $10 \%$. This remaining discrepancy can be attributed to finite volume corrections.

To address these finite volume corrections, we consider the chiral effective field theory study presented in Ref. [26]. Using this formalism, we estimate the finite volume corrections to our magnetic moment extractions at each pion mass. Our results corrected to their predicted infinitevolume values are presented in Fig. 18. We see that this correction brings our PEVA results much closer to the experimental value for the isovector magnetic moment. Performing the full chiral extrapolation as in Ref. [26], we find that the PEVA results are consistent with the experimental result, while the conventional results sit many standard deviations low. In this analysis, there is some instability in the extracted regulator parameter, due to the smaller number of ensembles considered here. To account 


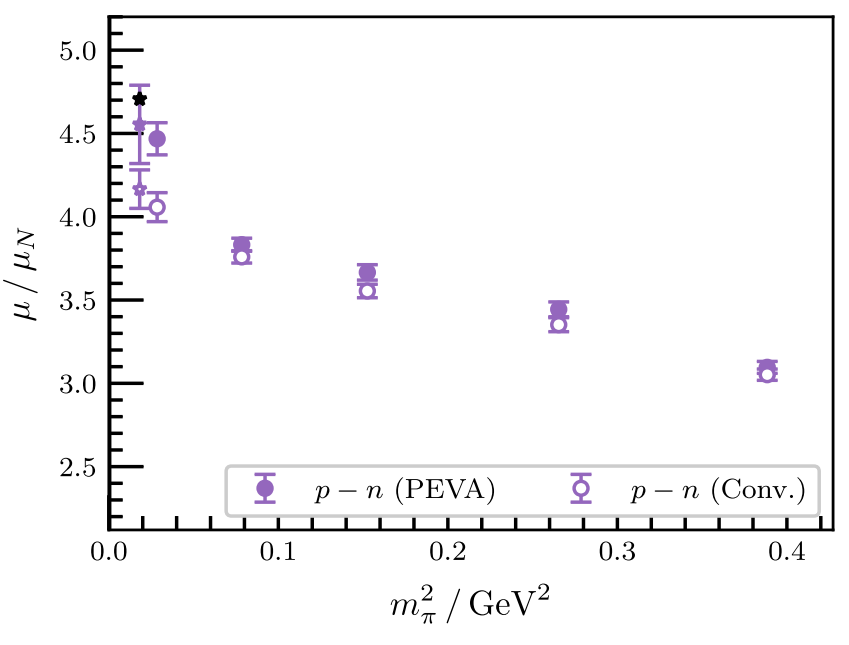

FIG. 18. Pion-mass dependence of the extracted magnetic moment for the isovector combination with finite-volume corrections from Ref. [26]. At the physical point, we present chiral extrapolations of the PEVA and conventional results with filled and open stars respectively. The PEVA result agrees well with the physical value of $4.70 \mu_{N}$ [19], represented by a black star.

for this, we have included in our analysis an additional systematic error not considered in the reference, arising from varying $m_{\pi, \max }^{2}$ from $0.39(1) \mathrm{GeV}$ to $0.27(1) \mathrm{GeV}$. Because the PEVA results show a much stronger chiral curvature, the extrapolation of these results is more sensitive to uncertainties in the regulator, as seen in its larger error bars.

These results clearly indicate that the magnetic moment extracted through the conventional analysis is significantly affected by opposite parity contaminations, resulting in incorrect results. The PEVA analysis allows us to remove these contaminations, bringing our results in line with experiment.

\section{CONCLUSION}

In this paper, we extended the parity-expanded variational analysis (PEVA) technique to the calculation of elastic form factors, and applied it to calculating the Sachs electric and magnetic form factors of the ground-state proton and neutron. This required inspection of the Dirac structure of the three point correlation function and careful selection of appropriate spinor projectors to extract the desired form factors with maximized signal.

Nucleon structure is a vibrant and rich field of study, and there have been investigations of the Sachs electric and magnetic form factors of the ground state nucleon spanning decades. In this paper we focused specifically on the application of the PEVA technique [1] to form factor calculations and on the systematic errors introduced by opposite-parity contaminations which may be present in conventional analyses.

We demonstrated the efficacy of variational analysis techniques in general, and PEVA specifically, at controlling excited-state contaminations in the electric form factor. Both the PEVA and conventional variational analysis show clear and clean plateaus, supporting previous work demonstrating the utility of variational analysis in calculating baryon matrix elements [21,22].

In the particular case of the magnetic form factor, we found evidence that the conventional analysis was contaminated by opposite-parity states. For the kinematics considered here, we observe $\sim 20 \%$ underestimation of the magnitude of the contributions to the magnetic form factor from the singly represented quark flavor at the lighter pion masses. The only difference in the interpolators is that opposite-parity contaminations can be addressed in our new PEVA approach. The difference indicates these contaminations are present in the standard variational approach. As the PEVA approach provides additional interpolator degrees of freedom to improve the ground state interpolating field, this is the improved interpolating field.

Further evidence of the improvement afforded by the PEVA approach is presented in Ref. [27], where we explored excited states of the nucleon at larger quark masses. Only the PEVA approach is able to resolve magnetic form factors in accord with constituent quark models. Quark models are renowned for capturing the qualitative features of baryon magnetic moments at larger quark masses.

These results indicate that existing calculations that do not take into account opposite-parity contaminations may be affected by systematic errors on the order of $20 \%$ at physical quark masses. As such, the PEVA technique is critical for precision measurements of the nucleon form factors.

By utilizing the PEVA technique and boosted-frame techniques, we are able to successfully extract the Sachs form factors of the ground-state nucleon at a range of $Q^{2}$ values. These extractions allow us to investigate the $Q^{2}$ dependence of these form factors. By taking ratios of the form factors, we are also able to extract the magnetic moments of both the ground-state proton and the groundstate neutron.

This paper has established the groundwork for applying the PEVA technique to calculating baryonic matrix elements. The applications for future research are broad. The techniques presented here could be applied directly to the examination of other nucleon observables or excited state observables. One possibility is to examine a matrix element that should vanish in the ground-state nucleon, where any nonzero value is evidence of excited state contamination. An extension is currently underway for the calculation of nucleon transition form factors.

A straightforward application of the calculations performed here, with extra statistics and a range of lattice spacings and volumes to quantify systematic errors, could provide state-of-the-art ab-initio determinations of the nucleon electromagnetic form factors. Such a study should also be able to fully quantify the difference between PEVA and conventional results at the physical point and confirm 
the disagreement between the conventional magnetic moment extractions and the physical results.

Our results indicate that excited-state effects can be much worse in three-point functions than two-point functions. The contrast between the good agreement between PEVA and conventional extractions of the ground state mass and the disagreement between extractions of the magnetic form factor indicate that a plateau in the two-point correlator is thus insufficient evidence to be confident that the three-point correlator will be free of excited-state contaminations. Future calculations can consider a range of current insertion times following the onset of groundstate dominance in the two-point function to quantitatively assess excited-state systematics.

An approach to applying PEVA to spin-3/2 states is under consideration, allowing the issue of parity mixing to be addressed for a wider range of baryonic states. Such an extension would also allow for the inclusion of spin-3/2 states in the variational analysis for the nucleon, addressing systematic errors that may arise from the mixing of eigenstates of total angular momentum in moving frames. For a fully comprehensive study of excited state contaminations of the ground state nucleon multiparticle states should also be considered. Rigorously treating such states requires nonlocal interpolators and this would be a challenging but worthwhile avenue for further study, as it could simultaneously give additional insight into the nature of nucleon resonances. A comprehensive study addressing all of these sources of excited state contaminations could look at how strong the effects of each contaminant are and determine which states are most responsible for errors in the matrix elements extracted by conventional techniques.

A particularly interesting development in the field that could benefit greatly from the application of the PEVA technique is the computation of nonforward matrix elements from two point correlation functions via the Feynman-Hellmann theorem [28].

\section{ACKNOWLEDGMENTS}

This research was undertaken with the assistance of resources from the Phoenix HPC service at the University of Adelaide, the National Computational Infrastructure (NCI), which is supported by the Australian Government, and by resources provided by the Pawsey Supercomputing Centre with funding from the Australian Government and the Government of Western Australia. These resources were provided through the National Computational Merit Allocation Scheme and the University of Adelaide partner share. This research is supported by the Australian Research Council through Grants No. DP140103067, No. DP150103164, and No. LE160100051.
[1] F. M. Stokes, W. Kamleh, D. B. Leinweber, M. S. Mahbub, B. J. Menadue, and B. J. Owen, Parity-expanded variational analysis for nonzero momentum, Phys. Rev. D 92, 114506 (2015).

[2] K. C. Bowler, R. D. Kenway, L. Lellouch, J. Nieves, O. Oliveira, D. G. Richards, C. T. Sachrajda, N. Stella, and P. Ueberholz (UKQCD Collaboration), First lattice study of semileptonic decays of $\Lambda_{b}$ and $\Xi_{b}$ baryons, Phys. Rev. D 57, 6948 (1998).

[3] C. Michael, Adjoint sources in lattice gauge theory, Nucl. Phys. B259, 58 (1985).

[4] M. Luscher and U. Wolff, How to calculate the elastic scattering matrix in two-dimensional quantum field theories by numerical simulation, Nucl. Phys. B339, 222 (1990).

[5] G. Martinelli, C. T. Sachrajda, and A. Vladikas, A study of "improvement" in lattice QCD, Nucl. Phys. B358, 212 (1991).

[6] S. Boinepalli, D. B. Leinweber, A. G. Williams, J. M. Zanotti, and J. B. Zhang, Precision electromagnetic structure of octet baryons in the chiral regime, Phys. Rev. D 74, 093005 (2006).

[7] D. B. Leinweber, R. M. Woloshyn, and T. Draper, Electromagnetic structure of octet baryons, Phys. Rev. D 43, 1659 (1991).
[8] S. Aoki, K. Ishikawa, N. Ishizuka, T. Izubuchi, D. Kadoh, K. Kanaya, Y. Kuramashi, Y. Namekawa, M. Okawa, Y. Taniguchi, A. Ukawa, N. Ukita, and T. Yoshie (PACS-CS Collaboration), $2+1$ flavor lattice QCD toward the physical point, Phys. Rev. D 79, 034503 (2009).

[9] M. G. Beckett, B. Joo, C. M. Maynard, D. Pleiter, O. Tatebe, and T. Yoshie, Building the International Lattice Data Grid, Comput. Phys. Commun. 182, 1208 (2011).

[10] M. S. Mahbub, W. Kamleh, D. B. Leinweber, P. J. Moran, and A.G. Williams, Structure and flow of the nucleon eigenstates in lattice QCD, Phys. Rev. D 87, 094506 (2013).

[11] S. Gusken, A study of smearing techniques for hadron correlation functions, Nucl. Phys. B, Proc. Suppl. 17, 361 (1990).

[12] C. Morningstar and M.J. Peardon, Analytic smearing of $S U(3)$ link variables in lattice QCD, Phys. Rev. D 69, 054501 (2004).

[13] C. W. Bernard, T. Draper, G. Hockney, and A. Soni, Calculation of weak matrix elements: Some technical aspects, Lattice Gauge Theory. NATO ASI Series (Springer, Boston, 1985), pp. 199-207, DOI: 10.1007/978-1-4613-2231-3_17.

[14] B. Blossier, M. Della Morte, G. von Hippel, T. Mendes, and R. Sommer (ALPHA collaboration), On the generalized eigenvalue method for energies and matrix elements in lattice field theory, J. High Energy Phys. 04 (2009) 094. 
[15] J. M. M. Hall, D. B. Leinweber, B. J. Owen, and R. D. Young, Finite-volume corrections to charge radii, Phys. Lett. B 725, 101 (2013).

[16] R. Pohl, R. Gilman, G. A. Miller, and K. Pachucki, Muonic hydrogen and the proton radius puzzle, Annu. Rev. Nucl. Part. Sci. 63, 175 (2013).

[17] C. E. Carlson, The proton radius puzzle, Prog. Part. Nucl. Phys. 82, 59 (2015).

[18] A. Antognini et al., Proton structure from the measurement of $2 \mathrm{~S}-2 \mathrm{P}$ transition frequencies of muonic hydrogen, Science 339, 417 (2013).

[19] P. J. Mohr, D. B. Newell, and B. N. Taylor, CODATA recommended values of the fundamental physical constants: 2014, Rev. Mod. Phys. 88, 035009 (2016).

[20] A. Beyer, L. Maisenbacher, A. Matveev, R. Pohl, K. Khabarova, A. Grinin, T. Lamour, D. C. Yost, T. W. Hänsch, N. Kolachevsky, and T. Udem, The Rydberg constant and proton size from atomic hydrogen, Science 358, 79 (2017).

[21] B. J. Owen, J. Dragos, W. Kamleh, D. B. Leinweber, M. S. Mahbub, B. J. Menadue, and J. M. Zanotti, Variational approach to the calculation of $g_{A}$, Phys. Lett. B 723, 217 (2013).

[22] J. Dragos, R. Horsley, W. Kamleh, D. B. Leinweber, Y. Nakamura, P. E. L. Rakow, G. Schierholz, R. D. Young, and J.M. Zanotti, Nucleon matrix elements using the variational method in lattice QCD, Phys. Rev. D 94, 074505 (2016).

[23] M. J. Savage, The magnetic moments of the octet baryons in quenched chiral perturbation theory, Nucl. Phys. A700, 359 (2002).

[24] D. B. Leinweber, Quark contributions to baryon magnetic moments in full, quenched, and partially quenched QCD, Phys. Rev. D 69, 014005 (2004).

[25] V. Punjabi et al., Proton elastic form factor ratios to $Q^{2}=$ $3.5 \mathrm{GeV}^{2}$ by polarization transfer, Phys. Rev. C 71, 055202 (2005); Erratum 71, 069902(E) (2005).

[26] J. M. M. Hall, D. B. Leinweber, and R. D. Young, Chiral extrapolations for nucleon magnetic moments, Phys. Rev. D 85, 094502 (2012).

[27] W. Kamleh, D. Leinweber, Z.-w. Liu, F. Stokes, A. Thomas, S. Thomas, and J.-j. Wu, Structure of the nucleon and its excitations, EPJ Web Conf. 175, 06019 (2018).

[28] A. J. Chambers, J. Dragos, R. Horsley, Y. Nakamura, H. Perlt, D. Pleiter, P. Rakow, G. Schierholz, A. Schiller, K. Somfleth, H. Stuben, R. Young, and J. Zanotti (UKQCD, QCDSF, and CSSM Collaborations), Electromagnetic form factors at large momenta from lattice QCD, Phys. Rev. D 96, 114509 (2017). 